\title{
Some Absolute Continuity Relationships for Certain Anticipative Transformations of Geometric Brownian Motions
}

\author{
By
}

Catherine Donati-Martin, Hiroyuki Matsumoto** and Marc YoR***

\begin{abstract}
We present some absolute continuity relationships between the probability laws of a geometric Brownian motion $e^{(\mu)}=\left\{e_{t}^{(\mu)}, t \geqq 0\right\}$ and its images by certain transforms $T_{\alpha}$ involving $e^{(\mu)}$ and its quadratic variation $\left\{\left\langle e^{(\mu)}\right\rangle_{t}, t \geqq 0\right\}$. These results are derived from, and shown to be closely related to, our previous results about the generalized Dufresne's identity and the exponential type extensions of Pitman's $2 M-X$ theorem for $X$, a Brownian motion with constant drift $\mu$, and its one-sided supremum $M$. These absolute continuity results are then shown to be particular cases of those by Ramer-Kusuoka for non-linear transformations of the Wiener space and by Buckdahn-Föllmer for solutions of certain stochastic differential equations with anticipative drifts.
\end{abstract}

\section{$\S 1 . \quad$ Introduction}

1.1. Let $B=\left\{B_{t}, t \geqq 0\right\}$ denote a one-dimensional Brownian motion starting from 0 and set $A_{t}=\int_{0}^{t} \exp \left(2 B_{s}\right) d s$; more generally, for $\mu \in \mathbf{R}$, we consider the Brownian motion $B^{(\mu)}$ with constant drift $\mu$ and the corresponding exponential functional defined by

$$
B_{t}^{(\mu)}=B_{t}+\mu t \quad \text { and } \quad A_{t}^{(\mu)} \equiv A_{t}\left(B^{(\mu)}\right)=\int_{0}^{t} \exp \left(2 B_{s}^{(\mu)}\right) d s, \quad t \geqq 0,
$$

Communicated by Y. Takahashi, September 13, 2000.

2000 Mathematics Subject Classification(s): 60J65.

* Laboratoire de Statistique et Probabilités, Université Paul Sabatier, 118, route de Narbonne, 31062 Toulouse Cedex 04, France.

** School of Informatics and Sciences, Nagoya University, Chikusa-ku, Nagoya 464-8601, Japan.

*** Laboratoire de Probabilités, Université Pierre et Marie Curie, 4, Place Jussieu, Tour 56, F-75252 Paris Cedex 05, France. 
respectively.

In a series of papers $([12],[13],[14])$, the main results of which have been summarized in [11], we obtained the following:

(a) The identity in law

$$
\left\{\frac{1}{A_{t}^{(-\mu)}}, t \geqq 0\right\} \stackrel{\text { (law) }}{=}\left\{\frac{1}{A_{t}^{(\mu)}}+\frac{1}{\tilde{A}_{\infty}^{(-\mu)}}, t \geqq 0\right\}
$$

holds for every $\mu>0$, where $\tilde{A}_{\infty}^{(-\mu)}$ is a copy of $A_{\infty}^{(-\mu)}=\lim _{t \rightarrow \infty} A_{t}^{(-\mu)}$, independent of $\left\{A_{t}^{(\mu)}, t \geqq 0\right\}$. This result extends that of Dufresne [7], who obtained the identity in law for any fixed time. Moreover one has

$$
A_{\infty}^{(-\mu)} \stackrel{\text { (law) }}{=} \frac{1}{2 \gamma_{\mu}}
$$

for a $\operatorname{Gamma}(\mu)$ random variable $\gamma_{\mu}([6],[20])$.

(b) The stochastic processes $\left\{Z_{t}^{(\mu)} \equiv \exp \left(-B_{t}^{(\mu)}\right) A_{t}^{(\mu)}, t \geqq 0\right\}$ and $\left\{Z_{t}^{(-\mu)} \equiv\right.$ $\left.\exp \left(-B_{t}^{(-\mu)}\right) A_{t}^{(-\mu)}, t \geqq 0\right\}$ have the same distribution, precisely that of a diffusion process with infinitesimal generator

$$
\frac{1}{2} z^{2} \frac{d^{2}}{d z^{2}}+\left\{\left(\frac{1}{2}-\mu\right) z+\left(\frac{K_{1+\mu}}{K_{\mu}}\right)\left(\frac{1}{z}\right)\right\} \frac{d}{d z}
$$

where $K_{\nu}$ is the usual modified Bessel (Macdonald) function. As discussed in [11], [13] and [14], this result should be considered as an extension and an analogue of Pitman's celebrated $2 M-X$ theorem.

(c) For any fixed $t>0$, the conditional probability law of $e_{t}^{(\mu)} \equiv \exp \left(B_{t}^{(\mu)}\right)$ given $\mathcal{Z}_{t}^{(\mu)} \equiv \sigma\left\{Z_{s}^{(\mu)}, s \leqq t\right\}$ is a generalized inverse Gaussian (GIG) distribution; more precisely, it is given by

$$
\begin{aligned}
P\left(e_{t}^{(\mu)}\right. & \left.\in d x \mid \mathcal{Z}_{t}^{(\mu)}, Z_{t}^{(\mu)}=z\right) \\
& =\frac{1}{2 K_{\mu}(1 / z)} x^{\mu-1} \exp \left(-\frac{1}{2 z}\left(x+\frac{1}{x}\right)\right) d x, \quad x>0 .
\end{aligned}
$$

The identity (1.4) has played a key role in our previous proof of the result mentioned in (b) above and, in particular, it shows that $\mathcal{Z}_{t}^{(\mu)}$ is strictly included in $\mathcal{B}_{t} \equiv \sigma\left\{B_{s}, s \leqq t\right\}$. Moreover, one has $\mathcal{B}_{t}=\mathcal{Z}_{t}^{(\mu)} \vee \sigma\left\{B_{t}\right\}$.

Before we discuss any further, we emphasize that we found the Lamperti relationship

$$
e_{t}^{(\mu)}=R_{A_{t}^{(\mu)}}^{(\mu)}, \quad t \geqq 0
$$


where $\left\{R_{u}^{(\mu)}, u \geqq 0\right\}$ is a Bessel process of index $\mu$ or of dimension $\delta=2(1+\mu)$, starting from 1 , to be an essential tool in our derivation of the above mentioned results.

1.2. The origin of the present work has been our desire to give a more direct proof of (1.4) than in [13], [14], using only (1.1) and the following well-known Doob $h$-transform relation between the respective distributions $P^{(\mu)}$ and $P^{(-\mu)}$ of $B^{(\mu)}$ and $B^{(-\mu)}$ considered on $C\left(\mathbf{R}_{+}, \mathbf{R}\right)$ :

$$
\left.P^{(\mu)}\right|_{\mathcal{F}_{t}}=\left.\exp \left(2 \mu X_{t}\right) P^{(-\mu)}\right|_{\mathcal{F}_{t}}
$$

where $\mathcal{F}_{t}=\sigma\left\{X_{s}, s \leqq t\right\}$ and $X_{t}=X_{t}(w)=w(t)$ for $w \in C\left(\mathbf{R}_{+}, \mathbf{R}\right)$.

For this purpose we show the following relationship, which is the main new result in this work and which is of interest by itself.

Theorem 1.1. Let $\mu \in \mathbf{R}, \alpha \in \mathbf{R}_{+}$and $F: C\left(\mathbf{R}_{+}, \mathbf{R}\right) \rightarrow \mathbf{R}_{+}$be an adapted functional. Then, for every $t>0$, it holds that

$$
E\left[F\left(B_{s}^{(\mu)}-\log \left(1+\alpha A_{s}^{(\mu)} / e_{t}^{(\mu)}\right), s \leqq t\right)\right]=E\left[F\left(B_{s}^{(\mu)}, s \leqq t\right) \Gamma_{\alpha}^{(\mu)}\left(e_{t}^{(\mu)}, Z_{t}^{(\mu)}\right)\right],
$$

where $\Gamma_{\alpha}^{(\mu)}(x, z)=(1+\alpha z)^{\mu} \Gamma_{\alpha}(x, z)$ and

$$
\Gamma_{\alpha}(x, z)=\exp \left(-\frac{\alpha}{2}\left(x-\frac{1}{(1+\alpha z) x}\right)\right) \text {. }
$$

It may be convenient to present the following variant of the identity (1.6).

Corollary 1.2. For every $t>0$, it holds that

$$
E\left[F\left(B_{s}^{(\mu)}-\log \left(1+\alpha A_{s}^{(\mu)} / e_{t}^{(\mu)}\right), s \leqq t\right) \tilde{\Gamma}_{\alpha}^{(\mu)}\left(e_{t}^{(\mu)}, Z_{t}^{(\mu)}\right)\right]=E\left[F\left(B_{s}^{(\mu)}, s \leqq t\right)\right],
$$

where $\tilde{\Gamma}_{\alpha}^{(\mu)}(x, z)=\Gamma_{\alpha}^{(-\mu)}(1 / x, z)$.

The following consequences of Theorem 1.1 are easily obtained; in particular, the next result may be considered as a companion to formula (1.1), but the reader should beware that it involves only $A^{(\mu)}$ and not the pair $\left(A^{(\mu)}, A^{(-\mu)}\right)$.

Corollary 1.3. Under the same notations as in Theorem 1.1, the following relationship holds:

$$
E\left[F\left(\frac{1}{A_{s}^{(\mu)}}+\left(\alpha / e_{t}^{(\mu)}\right), s \leqq t\right)\right]=E\left[F\left(\frac{1}{A_{s}^{(\mu)}}, s \leqq t\right) \Gamma_{\alpha}^{(\mu)}\left(e_{t}^{(\mu)}, Z_{t}^{(\mu)}\right)\right]
$$


Corollary 1.4. The identity (1.4) holds.

We shall give a detailed proof of Corollary 1.4 in Section 5 below.

1.3. We now comment on the title of the present paper. To do this, let us introduce the $T_{\alpha}$-transform of $B^{(\mu)}$ defined by

$$
T_{\alpha}\left(B^{(\mu)}\right)_{s} \equiv B_{s}^{(\mu)}-\log \left(1+\alpha A_{s}^{(\mu)}\right), \quad s \geqq 0 .
$$

Then we see that the expectation on the left hand side of (1.6) is that for $T_{\alpha / e_{t}^{(\mu)}}\left(B^{(\mu)}\right)_{s}, s \leqq t$, and it is natural to call such a transform anticipative since, at time $s$, it involves $B_{t}^{(\mu)}$ for $t \geqq s$.

This remark being made, Theorem 1.1 is some kind of Maruyama-Girsanov theorem involving an anticipative transform of $B^{(\mu)}$. Note also that we have an unusual absolutely continuity relationship between the laws of $\left\{1 / A_{s}^{(\mu)}, s \leqq t\right\}$ and its (random) translate $\left\{1 / A_{s}^{(\mu)}+\alpha / e_{t}^{(\mu)}, s \leqq t\right\}$ given by (1.8).

1.4. It is now natural to look for some analogue of Theorem 1.1 which involves the simpler transform $T_{\alpha}$ instead of $T_{\alpha / e_{t}^{(\mu)}}$. This is the content of the following.

Theorem 1.5. For any adapted non-negative functional $G$ and any $\mu \geqq$ 0 , it holds that

$$
E\left[G\left(T_{\alpha}\left(B^{(\mu)}\right)_{s}, s \leqq t\right)\right]=E\left[G\left(B_{s}^{(\mu)}, s \leqq t\right) \Delta_{\alpha}^{(\mu)}\left(e_{t}^{(\mu)}, \alpha A_{t}^{(\mu)}\right)\right],
$$

where $\Delta_{\alpha}^{(\mu)}(v, u)=(1-u)^{-\mu} \Delta_{\alpha}(v, u)$ and

$$
\Delta_{\alpha}(v, u)=\frac{\mathbf{1}_{\{u<1\}}}{1-u} \exp \left(-\frac{\alpha}{2}\left(\frac{v^{2}}{1-u}-1\right)\right) .
$$

Moreover, there is an analogue of the Lamperti relation:

$$
\exp \left(T_{\alpha}\left(B^{(\mu)}\right)_{t}\right)=R_{A\left(T_{\alpha}\left(B^{(\mu)}\right)\right)_{t}}^{(\mu, 1 / \alpha)}
$$

where $\left\{R_{u}^{(\mu, 1 / \alpha)}, u \leqq 1 / \alpha\right\}$ is a Bessel bridge with dimension $\delta=2(1+\mu)$, starting from 1 at time 0 and conditioned to be at 0 at time $1 / \alpha$.

Remark 1.1. It should be mentioned that the law of $\left\{T_{\alpha}(B)_{s}, s \leqq t\right\}$ cannot be equivalent to that of $B$ because

$$
\frac{1}{A\left(T_{\alpha}(B)\right)_{t}}=\frac{1}{A(B)_{t}}+\alpha, \quad \text { hence } \quad A\left(T_{\alpha}(B)\right)_{t}<\frac{1}{\alpha} .
$$


This also implies that the local martingale $\left\{M_{t}, t \geqq 0\right\}$ given by

$$
M_{t}=\exp \left(\int_{0}^{t} \frac{\alpha \exp \left(2 B_{s}\right)}{1+\alpha A_{s}} d B_{s}-\frac{1}{2} \int_{0}^{t}\left(\frac{\alpha \exp \left(2 B_{s}\right)}{1+\alpha A_{s}}\right)^{2} d s\right)
$$

is not a martingale. In fact, if it were a martingale under the probability measure $P$, then, denoting by $Q$ the probability measure such that $\left.Q\right|_{\mathcal{B}_{t}}=$ $\left.M_{t} \cdot P\right|_{\mathcal{B}_{t}}$ and noting that $\left\{T_{\alpha}(B)_{t}, t \geqq 0\right\}$ is a Brownian motion under $Q$, we would have $P\left(A(B)_{t}<1 / \alpha\right)=Q\left(A\left(T_{\alpha}(B)\right)_{t}<1 / \alpha\right)=1$ for all $t$, which is wrong.

1.5. We now compare Theorems 1.1 and 1.5 from the point of view of changes of probability measures. Formula (1.9) in Theorem 1.5 looks like a particular case of the Maruyama-Girsanov theorem (see Remark 1.1 above), whereas Theorem 1.1 or Corollary 1.2 is a particular case of the Ramer-Kusuoka formula ([17], [9]) for anticipative transforms of Brownian motion.

In fact, applying the Ramer-Kusuoka formula, we have the following: if $\xi=\left\{\xi_{t}, t \geqq 0\right\}$ denotes the solution of

$$
\xi_{t}=w(t)+\int_{0}^{t} k_{s}(\xi(w), w(1)) d s
$$

where $k$ only anticipates by the end point $w(1)$ of the Brownian path $\{w(t), 0 \leqq$ $t \leqq 1\}$, then, under some conditions, the probability law $P_{\xi}$ of $\xi$ is absolutely continuous with respect to the Wiener measure $P$ and the density is given by

$$
\frac{d P_{\xi}}{d P}=\left|d_{c}(D H)\right| \exp \left(\delta(H)-\frac{1}{2} \int_{0}^{1} H_{s}^{2} d s\right),
$$

where $H=\left\{H_{s}\right\}$ is given by $H_{s}=k_{s}\left(w, \eta_{1}(w)\right)$ for the inverse transform $\eta$ of $\xi, d_{c}(D H)$ is the Carleman-Fredholm determinant of the Malliavin derivative $D H$ and $\delta(H)$ is the Skorohod integral of $H$.

Moreover, Buckdahn-Föllmer [4] have proved the following specialization of (1.12): under some regularity and integrability conditions,

$$
\begin{aligned}
\frac{d P_{\xi}}{d P}=\mid 1 & +\left.\int_{0}^{1} k_{s}^{\prime}\left(w, \eta_{1}(w)\right) d s\right|^{-1} \\
& \times \exp \left(\int_{0}^{1} k_{s}\left(w, \eta_{1}(w)\right) d w(s)-\frac{1}{2} \int_{0}^{1} k_{s}\left(w, \eta_{1}(w)\right)^{2} d s\right)
\end{aligned}
$$

where $k_{s}^{\prime}(w, y)=\frac{d}{d y} k_{s}(w, y)$ and $\eta_{1}(w)$ denotes the value at time 1 of the inverse transform $\eta$ of $w \mapsto \xi(w)$. 
In Section 8 below, we shall show that

$$
\xi_{t}(w)=w(t)-\log \left(1+\alpha A(w)_{t} / e(w)_{1}\right), \quad t \leqq 1,
$$

is precisely the solution of an equation of the form (1.11) and that formula (1.6) (for $\mu=0$ and $t=1$ for simplicity) can be understood as a particular case of (1.13), which we call the Buckdahn-Föllmer formula. Moreover, using Theorem 1.1, we will show that, in this case, all terms on the right hand side of (1.12) are expressed in explicit form.

1.6. At this point of the discussion, we find it important to explain more precisely why we got interested in the complicated transform $T_{\alpha / e_{t}^{(\mu)}}$ rather than in the simpler $T_{\alpha}$. Indeed, as we already noticed in Proposition 2.4 in [12] (see also [13], Section 14), there is a variant and an extension of the identity (1.1), which is presented as follows: for any $t>0$, one has

$$
\begin{aligned}
& \left(\gamma_{\mu} \exp \left(-B_{t}^{(-\mu)}\right),\left\{B_{s}^{(-\mu)}, s \leqq t\right\}\right) \\
& \quad \stackrel{\text { law })}{=}\left(\gamma_{\mu} \exp \left(B_{t}^{(\mu)}\right),\left\{B_{s}^{(\mu)}-\log \left(1+2 \gamma_{\mu} A_{s}^{(\mu)}\right), s \leqq t\right\}\right),
\end{aligned}
$$

where, on both hand sides, $\mu$ is positive and $\gamma_{\mu}$ denotes a $\operatorname{Gamma}(\mu)$ random variable which is independent of $B^{(-\mu)}$ on the left hand side and of $B^{(\mu)}$ on the right hand side.

Now the conditional probability law of $\left\{B_{s}^{(\mu)}-\log \left(1+2 \gamma_{\mu} A_{s}^{(\mu)}\right), s \leqq t\right\}$ given $\gamma_{\mu} \exp \left(B_{t}^{(\mu)}\right)=y$ is obviously that of $\left\{T_{2 y \exp \left(-B_{t}^{(\mu)}\right)}\left(B^{(\mu)}\right)_{s}, s \leqq t\right\}$ given $\gamma_{\mu} \exp \left(B_{t}^{(\mu)}\right)=y$. A more detailed discussion is provided in Section 3 .

Another way to relate the studies of the stochastic processes $\left\{T_{\alpha / e_{t}^{(\mu)}}\left(B^{(\mu)}\right)_{s}, s \leqq t\right\}$ and $\left\{T_{\alpha}\left(B^{(\mu)}\right)_{s}, s \leqq t\right\}$ is to look for a condition on a non-negative random variable $X$ which ensures that the laws of $\left\{T_{X}\left(B^{(\mu)}\right)_{s}, s \leqq t\right\}$ and $\left\{B_{s}^{(\mu)}, s \leqq t\right\}$ are equivalent. Besides Theorem 1.1, which asserts that $X=\alpha \exp \left(-B_{t}^{(\mu)}\right)$ satisfies this property, we also find, as a consequence of either Theorem 1.1 or Theorem 1.5, that, if $X$ is independent of $B^{(\mu)}$ and if the law of $X$ is equivalent to the Lebesgue measure on $\mathbf{R}_{+}$, then $X$ also satisfies this property and we can write down the density. This question is treated at the end of Section 6 .

1.7. The rest of this paper is organized as follows. In Section 2 we discuss some properties and characterizations of the transform $T_{\alpha}$, which helps us considerably in our proofs of Theorems 1.1 and 1.5. In Section 3 we discuss the result (1.14) and its consequences. In Section 4 we prove Theorem 1.1. In Section 5 we show how the generalized inverse Gaussian distribution presented in (1.4) follows from Theorem 1.1. In Section 6 we prove Theorem 1.5 and we 
compare it to Theorem 1.1. In Section 7 we study the "pseudo" martingale properties of the density $\Gamma_{\alpha}^{(\mu)}\left(e_{t}^{(\mu)}, Z_{t}^{(\mu)}\right)$ featured in (1.6). This density is not a martingale with respect to the natural filtration $\mathcal{F}_{t} \equiv \sigma\left\{B_{s}^{(\mu)}, s \leqq t\right\}$, but it has some "similar" properties, hence we call it a pseudo martingale. In Section 8 we give the details of the relation of our work with the Ramer-Kusuoka and the Buckdahn-Föllmer formulae. Finally, Section 9 consists of several questions and remarks strongly motivated by Theorems 1.1 and 1.5.

\section{$\S 2$. The $T_{\alpha}$ Transforms}

2.1. Algebraic properties of $\boldsymbol{T}_{\boldsymbol{\alpha}}$. The studies made in the present paper and also in our previous papers [12], [13], [14] are best understood if we introduce the following transforms from $C\left(\mathbf{R}_{+}, \mathbf{R}\right)$ to itself: to a given continuous function $\phi: \mathbf{R}_{+} \rightarrow \mathbf{R}$, we associate $Z_{t}(\phi)=\exp (-\phi(t)) A_{t}(\phi)$, where $A_{t}(\phi)=\int_{0}^{t} \exp (2 \phi(s)) d s$, as well as $T_{\alpha}(\phi)_{t}=\phi(t)-\log \left(1+\alpha A_{t}(\phi)\right)$.

We then note the easily proven properties.

Proposition 2.1. For every $\phi \in C\left(\mathbf{R}_{+}, \mathbf{R}\right)$ and $\alpha>0$, one has
(i) $\frac{d}{d t}\left(\frac{1}{A_{t}(\phi)}\right)=-\frac{1}{Z_{t}(\phi)^{2}}$,
(ii) $\frac{1}{A_{t}\left(T_{\alpha}(\phi)\right)}=\frac{1}{A_{t}(\phi)}+\alpha, \quad t \geqq 0$,
(iii) $Z \circ T_{\alpha}=Z$,
(iv) $T_{\alpha} \circ T_{\beta}=T_{\alpha+\beta}$.

Proof. (i) and (ii) are immediate. (iii) is then deduced from (ii) by taking the derivatives of both hand sides in (ii) and by using (i). (iv) is now easily deduced since, as a consequence of (ii), $A \circ T_{\alpha} \circ T_{\beta}=A \circ T_{\alpha+\beta}$ obviously holds and $A$ is injective.

Remark 2.1. It is also possible to define the transform $T_{-\alpha}$, for $\alpha>$ 0 , at least on $T_{\alpha}\left(C\left(\mathbf{R}_{+}, \mathbf{R}\right)\right)$ since, if $\phi$ belongs to this subset of $C\left(\mathbf{R}_{+}, \mathbf{R}\right)$, $A_{t}(\phi)<1 / \alpha$, hence $\log \left(1-\alpha A_{t}(\phi)\right)$ is well-defined. Furthermore, one obtains (iv) $T_{-\alpha} \circ T_{\beta}=T_{\beta-\alpha}$ for $\beta \geqq \alpha$.

2.2. Characterization of $\boldsymbol{T}_{\boldsymbol{\alpha}}$. Given the essential role played by the transform $T_{\alpha}$ in the previous section, it is natural to look for some characterization of this transform. This is provided by the following.

Proposition 2.2. Consider two continuous functions $\phi, \tilde{\phi}: \mathbf{R}_{+} \rightarrow \mathbf{R}$ such that $\phi(0)=\tilde{\phi}(0), \phi(t)>\tilde{\phi}(t)$ and $Z_{t}(\phi)=Z_{t}(\tilde{\phi})$ for every $t>0$. Then there exists $\alpha>0$ such that $\tilde{\phi}(t)=\phi(t)-\log \left(1+\alpha A_{t}(\phi)\right)$, or, in other words, $\tilde{\phi}(t)=T_{\alpha}(\phi)_{t}, t>0$. 
Proof. Set $\Phi(t)=\phi(t)-\tilde{\phi}(t)$. Then our hypothesis entails

$$
\exp (\Phi(t)) \int_{0}^{t} \exp (2(\phi(u)-\Phi(u))) d u=\int_{0}^{t} \exp (2 \phi(u)) d u \equiv A_{t}(\phi), \quad t>0 .
$$

Therefore, setting $\psi_{t}=\exp (\Phi(t))$ (which is $C^{1}$ on $(0, \infty)$ ), we may write

$$
\int_{0}^{t} \frac{d A_{u}(\phi)}{\psi_{u}^{2}}=\frac{A_{t}(\phi)}{\psi_{t}}
$$

Taking differentials on both hand sides, we obtain

$$
\frac{d \psi_{t}}{\psi_{t}-1}=\frac{d A_{t}(\phi)}{A_{t}(\phi)}
$$

Note that, thanks to our hypothesis, the left hand side is well defined. Hence, there exists a constant $a \in \mathbf{R}$ such that $\log \left(\psi_{t}-1\right)=a+\log \left(A_{t}(\phi)\right), t>0$, and we obtain $\Phi(t)=\log \left(1+e^{a} A_{t}(\phi)\right)$.

Remark 2.2. We realize that our discussion of the transforms $T_{\alpha}$ and, in particular, the fact that $Z \circ T_{\alpha}=Z$ fulfills, in our set-up, the program developed by Beneš ([1], [2], [3]) of relating the non-injectivity of $Z$ and the loss of information it involves. Beneš' works are strongly motivated by Tsirel'son's celebrated stochastic differential equation (see also the discussion in [18], Chapter IX, Proposition 3.6).

2.3. Filtrations. We now consider the filtrations generated by $\left\{T_{\alpha}(B)_{s}, s \geqq\right.$ $0\}$ and the $\sigma$-fields of $\left\{T_{\alpha / e_{t}}(B)_{s}, s \leqq t\right\}$, since equalities of $\sigma$-fields or rather inclusions (corresponding to loss of information) play essential roles throughout our paper. As far as information is concerned, the stochastic processes $\left\{T_{\alpha}(B)_{s}, s \geqq 0\right\}$ and $B=\left\{B_{s}, s \geqq 0\right\}$ are equivalent. More precisely, we show the following.

Proposition 2.3. For any $\alpha>0$, the natural filtration $\mathcal{F}_{t}^{\alpha}$ of $\left\{T_{\alpha}(B)_{s}\right.$, $s \geqq 0\}$ is equal to that of $B$. Moreover, for any $\alpha>0$ and $t>0$, the $\sigma$-field $\mathcal{G}_{t}^{\alpha} \equiv \sigma\left\{T_{\alpha / e_{t}}(B)_{s}, s \leqq t\right\}$ is equal to $\mathcal{F}_{t} \equiv \sigma\left\{B_{s}, s \leqq t\right\}$, up to negligible sets.

Proof. The knowledge of $\left\{T_{\alpha}(B)_{s}, s \leqq t\right\}$ entails that of $Z_{s}\left(T_{\alpha}(B)\right)=$ $Z_{s}(B) \equiv A_{s} / e_{s}, s \leqq t$. Hence, by taking the logarithm and considering its difference from $T_{\alpha}(B)_{s}$, we see that the variable $\log \left(A_{s} /\left(1+\alpha A_{s}\right)\right)$ is $\mathcal{F}_{t}^{\alpha}-$ measurable. Consequently, for $s \leqq t, A_{s}$ and $Z_{s}$ are $\mathcal{F}_{t}^{\alpha}$-measurable. Therefore $e_{s}=\exp \left(B_{s}\right)=A_{s} / Z_{s}$ is also $\mathcal{F}_{t}^{\alpha}$-measurable, so that $\mathcal{F}_{t}^{\alpha}=\mathcal{F}_{t}$. 
Likewise, the stochastic process $\left\{Z_{s}(B), s \leqq t\right\}$ is measurable with respect to $\mathcal{G}_{t}^{\alpha}$; hence, the variable $B_{t}$ is $\mathcal{G}_{t}^{\alpha}$-measurable since $B_{t}=T_{\alpha / e_{t}}(B)_{t}+\log (1+$ $\left.\alpha Z_{t}(B)\right)$. Consequently, $\mathcal{F}_{t}=\mathcal{Z}_{t} \vee \sigma\left\{B_{t}\right\}$ is identical with $\mathcal{G}_{t}^{\alpha}$.

\section{§3. Amplification of the Generalized Dufresne's Identity (1.1)}

3.1. A new derivation of (1.1). The identity (1.14) presented in the Introduction is an extension of the identity (1.1): indeed, the identity in law between the second members on both hand sides of (1.14) tells us

$$
B^{(-\mu)} \stackrel{(\text { law })}{=} T_{2 \gamma_{\mu}}\left(B^{(\mu)}\right)
$$

which is equivalent to (1.1). Here we show that, conversely, (1.14) may be derived from (1.1).

For this purpose we remark that the left hand side of (1.14) may be represented as

$$
\begin{aligned}
& (3.1)\left(\frac{\exp \left(-B_{t}^{(-\mu)}\right)}{\int_{t}^{\infty} \exp \left(2\left(B_{u}^{(-\mu)}-B_{t}^{(-\mu)}\right)\right) d u},\left\{B_{s}^{(-\mu)}, s \leqq t\right\}\right) \\
& \stackrel{\text { (law) }}{=}\left(\frac{\exp \left(-\left(B_{t}^{(\mu)}-\log \left(1+2 \gamma_{\mu} A_{t}^{(\mu)}\right)\right)\right)}{\Delta},\left\{B_{s}^{(\mu)}-\log \left(1+2 \gamma_{\mu} A_{t}^{(\mu)}\right), s \leqq t\right\}\right),
\end{aligned}
$$

where

$$
\Delta=\int_{t}^{\infty} \frac{\exp \left(2\left(B_{u}^{(\mu)}-\log \left(1+2 \gamma_{\mu} A_{u}^{(\mu)}\right)\right)\right)}{\exp \left(2\left(B_{t}^{(\mu)}-\log \left(1+2 \gamma A_{t}^{(\mu)}\right)\right)\right)} d u
$$

and we have used the relationship $\left\{B_{s}^{(-\mu)}, s \geqq 0\right\} \stackrel{(\text { law })}{=}\left\{B_{s}^{(\mu)}-\log \left(1+2 \gamma_{\mu} A_{s}^{(\mu)}\right)\right.$, $s \geqq 0\}$ which follows from (1.1). Then it is easy to show

$$
\frac{\exp \left(-\left(B_{t}^{(\mu)}-\log \left(1+2 \gamma_{\mu} A_{t}^{(\mu)}\right)\right)\right)}{\Delta}=2 \gamma_{\mu} \exp \left(B_{t}^{(\mu)}\right)
$$

which finally yields (1.14).

3.2. Some consequences of (1.14). The identity in law (1.14) being derived anew, we make a list of some of its consequences, in particular, in order to explain our interest in the transform $T_{\alpha / e_{t}^{(\mu)}}$, where $e_{t}^{(\mu)}=\exp \left(B_{t}^{(\mu)}\right)$.

Proposition 3.1. $\quad$ Let $\mu>0$ and let $\gamma_{\mu}$ denote a Gamma $(\mu)$ random variable, independent of $B$. Then, for any non-negative adapted functional $F$ 
and for any $y>0$, the following quantities are equal:

(i) $E\left[F\left(B_{s}^{(\mu)}-\log \left(1+2 \gamma_{\mu} A_{s}^{(\mu)}\right), s \leqq t\right) \mid 2 \gamma_{\mu} e_{t}^{(\mu)}=y\right]$,

(ii) $\left(E\left[\exp \left(-\frac{y}{2 e_{t}}\right)\right]\right)^{-1} E\left[F\left(B_{s}-\log \left(1+y A_{s} / e_{t}\right), s \leqq t\right) \exp \left(-\frac{y}{2 e_{t}}\right)\right]$,

(iii) $E\left[F\left(B_{s}^{(-\mu)}, s \leqq t\right) \mid 2 \gamma_{\mu} / e_{t}^{(-\mu)}=y\right]$,

(iv) $E\left[\left.F\left(B_{s}^{(-\mu)}, s \leqq t\right)\right|^{+} Z_{t}^{(-\mu)}=1 / y\right]$,

where $^{+} Z_{t}^{(-\mu)}=\exp \left(-B_{t}^{(-\mu)}\right) \int_{t}^{\infty}\left(e_{s}^{(-\mu)}\right)^{2} d s$.

Proof. Let us denote $L_{s, t}^{(\mu)}(y)=B_{s}^{(\mu)}-\log \left(1+y A_{s}^{(\mu)} / e_{t}^{(\mu)}\right)$. Then we have

$$
\begin{aligned}
& E\left[F\left(L_{s, t}^{(\mu)}\left(2 \gamma_{\mu} e_{t}^{(\mu)}\right), s \leqq t\right) f\left(2 \gamma_{\mu} e_{t}^{(\mu)}\right)\right] \\
& =\frac{1}{\Gamma(\mu)} \int_{0}^{\infty} x^{\mu-1} e^{-x} E\left[F\left(L_{s, t}^{(\mu)}\left(2 x e_{t}^{(\mu)}\right), s \leqq t\right) f\left(2 x e_{t}^{(\mu)}\right)\right] d x \\
& =\frac{1}{\Gamma(\mu)} E\left[\int_{0}^{\infty} \frac{y^{\mu-1} \exp \left(-y / 2 e_{t}^{(\mu)}\right)}{\left(2 e_{t}^{(\mu)}\right)^{\mu}} F\left(L_{s, t}^{(\mu)}(y), s \leqq t\right) f(y)\right] d y \\
& =\frac{\exp \left(-\mu^{2} t / 2\right)}{2^{\mu} \Gamma(\mu)} \int_{0}^{\infty} y^{\mu-1} E\left[\exp \left(-y / 2 e_{t}\right) F\left(L_{s, t}^{(0)}(y), s \leqq t\right)\right] f(y) d y
\end{aligned}
$$

where the second equality is obtained with the help of the Cameron-Martin relationship between $B$ and $B^{(\mu)}$. The equality between the first and the last quantities in the preceding sequence of equalities yields the following two results:

$$
P\left(2 \gamma_{\mu} e_{t}^{(\mu)} \in d y\right)=e^{-\mu^{2} t / 2} \frac{y^{\mu-1}}{2^{\mu} \Gamma(\mu)} E\left[\exp \left(-\frac{y}{2 e_{t}}\right)\right] d y
$$

and

$$
\begin{aligned}
& E\left[F\left(L_{s, t}^{(\mu)}\left(2 \gamma_{\mu} e_{t}^{(\mu)}\right), s \leqq t\right) \mid 2 \gamma_{\mu} e_{t}^{(\mu)}=y\right] \\
& =\left(E\left[\exp \left(-\frac{y}{2 e_{t}}\right)\right]\right)^{-1} E\left[F\left(L_{s, t}^{(0)}(y), s \leqq t\right) \exp \left(-\frac{y}{2 e_{t}}\right)\right] .
\end{aligned}
$$

The identity (3.2) is precisely the equality between (i) and (ii) in the proposition. The equality between (i) and (iii) follows immediately from (1.14). Finally, the equality between (iii) and (iv) follows from the explanation given above in Subsection 3.1 for (1.14). 


\section{§4. Proof of Theorem 1.1}

4.1. Proof of Theorem 1.1. We now engage ourselves truly into a proof of Theorem 1.1, which we shall decompose into three steps.

Step 1. To deduce from (1.1) and (1.5) the identity

$$
E\left[\exp \left(-\eta e_{t}\right) F\left(\frac{1}{A_{s}}, s \leqq t\right)\right]=E\left[\exp \left(-\eta / e_{t}\right) F\left(\frac{1}{A_{s}}+2 \eta / e_{t}, s \leqq t\right)\right]
$$

for every $\eta>0$. From Proposition 2.1, we see that this identity is equivalent to the following:

$$
E\left[\exp \left(-\eta e_{t}\right) F\left(B_{s}, s \leqq t\right)\right]=E\left[\exp \left(-\eta / e_{t}\right) F\left(T_{2 \eta / e_{t}}(B)_{s}, s \leqq t\right)\right]
$$

Step 2. To deduce from (4.2) the identity (1.6) for $\mu=0$.

Step 3. To prove (1.6) for a general $\mu$.

4.2. Proof of Step 2. a) We proceed to Step 2, assuming that Step 1 has already been performed. Replacing $F$ on the right hand side of (4.2) by $F \exp \left(\eta e_{t}\right)$, we have

$$
\begin{array}{r}
E\left[F\left(B_{s}, s \leqq t\right)\right]=E\left[\exp \left(-\eta / e_{t}\right) \exp \left(\eta \exp \left(B_{t}-\log \left(1+2 \eta A_{t} / e_{t}\right)\right)\right)\right. \\
\left.\times F\left(T_{2 \eta / e_{t}}(B)_{s}, s \leqq t\right)\right],
\end{array}
$$

which, replacing $2 \eta$ by $\alpha$, is equivalent to

$$
E\left[F\left(B_{s}, s \leqq t\right)\right]=E\left[\tilde{\Gamma}_{\alpha}\left(e_{t}, Z_{t}\right) F\left(T_{\alpha / e_{t}}(B)_{s}, s \leqq t\right)\right]
$$

where

$$
\tilde{\Gamma}_{\alpha}(x, z)=\exp \left(\frac{\alpha}{2}\left(\frac{x}{1+\alpha z}-x^{-1}\right)\right) .
$$

We note that (4.3) is precisely (1.7) for $\mu=0$ and that the general case is obtained with the help of the Cameron-Martin formula, just as we do in Step 3.

b) In order to derive (1.6) for $\mu=0$ from (4.3), we seek a function $\varphi$ on $\mathbf{R} \times \mathbf{R}_{+}$such that

$$
E\left[\varphi\left(B_{t}, A_{t}\right) F\left(B_{s}, s \leqq t\right)\right]=E\left[F\left(T_{\alpha / e_{t}}(B)_{s}, s \leqq t\right)\right]
$$


Thus, in order that (4.4) is satisfied, we need

$$
\varphi\left(B_{t}-\log \left(1+\alpha Z_{t}\right), \frac{A_{t}}{1+\alpha Z_{t}}\right) \tilde{\Gamma}_{\alpha}\left(e_{t}, Z_{t}\right)=1
$$

because we have from the definitions of $T_{\beta}, A$ and $Z$

$$
\int_{0}^{t} \exp \left(2 T_{\alpha / e_{t}}(B)_{s}\right) d s=\int_{0}^{t} \frac{\left(e_{s}\right)^{2}}{\left(1+\alpha\left(e_{t}\right)^{-1} A_{s}\right)^{2}} d s=\frac{A_{t}}{1+\alpha Z_{t}}
$$

This can be accomplished without difficulty and it is sufficient to set

$$
\varphi(b, u)=\exp \left(-\frac{\alpha}{2}\left(e^{b}-\frac{1}{e^{b}+\alpha u}\right)\right) .
$$

Noting that

$$
\varphi\left(B_{t}, A_{t}\right)=\exp \left(-\frac{\alpha}{2}\left(e_{t}-\frac{1}{\left(1+\alpha Z_{t}\right) e_{t}}\right)\right)=\Gamma_{\alpha}\left(e_{t}, Z_{t}\right),
$$

we complete the proof of (1.6) for $\mu=0$.

4.3. Proof of Step 3. For this purpose, we apply the Cameron-Martin theorem and replace, in formula (1.6) for $\mu=0$, the functional $F(\phi(s), s \leqq t)$ by $F(\phi(s), s \leqq t) \exp (\mu \phi(t))$. Then, using $Z\left(T_{\alpha / e_{t}}(B)\right)_{t}=Z(B)_{t}$, we obtain

$$
\begin{aligned}
E\left[F \left(T_{\left.\left.\alpha / e_{t}^{(\mu)}\left(B^{(\mu)}\right)_{s}, s \leqq t\right)\right]}\right.\right. & =E\left[F\left(T_{\alpha / e_{t}}(B)_{s}, s \leqq t\right) e^{\mu B_{t}-\mu^{2} t / 2}\right] \\
& =E\left[F\left(B_{s}, s \leqq t\right) \Gamma_{\alpha}\left(e_{t}, Z_{t}\right)\left(1+\alpha Z_{t}\right)^{\mu} e^{\mu B_{t}-\mu^{2} t / 2}\right] \\
& =E\left[F\left(B_{t}^{(\mu)}, s \leqq t\right) \Gamma_{\alpha}^{(\mu)}\left(e_{t}^{(\mu)}, Z_{t}^{(\mu)}\right)\right]
\end{aligned}
$$

4.4. Proof of Step 1. At first we rewrite (1.1) in the equivalent form:

$$
E\left[F\left(\frac{1}{A_{s}^{(\mu)}}+\frac{1}{\tilde{A}_{\infty}^{(-\mu)}}, s \leqq t\right)\right]=E\left[F\left(\frac{1}{A_{s}^{(-\mu)}}, s \leqq t\right)\right] .
$$

Then, with the help of (1.2) and (1.5), the left hand side of (4.5) may be written as

$$
\begin{aligned}
\frac{1}{\Gamma(\mu)} \int_{0}^{\infty} \eta^{\mu-1} e^{-\eta} E\left[F\left(\frac{1}{A_{s}^{(\mu)}}+2 \eta, s \leqq t\right)\right] d \eta \\
=\frac{1}{\Gamma(\mu)} \int_{0}^{\infty} \eta^{\mu-1} e^{-\eta} E\left[F\left(\frac{1}{A_{s}^{(-\mu)}}+2 \eta, s \leqq t\right)\left(e_{t}^{(-\mu)}\right)^{2 \mu}\right] d \eta
\end{aligned}
$$

which is equal to $E\left[F\left(1 / A_{s}^{(-\mu)}, s \leqq t\right)\right]$. 
We then use the Cameron-Martin relationship to transform the last two expressions into related ones involving Brownian motion without drift. Thus we obtain

$$
\begin{aligned}
\int_{0}^{\infty} e^{-\eta} \eta^{\mu-1} E\left[F \left(\frac{1}{A_{s}}+\right.\right. & \left.2 \eta, s \leqq t)\left(e_{t}\right)^{\mu}\right] d \eta \\
& =\int_{0}^{\infty} e^{-\eta} \eta^{\mu-1} E\left[F\left(\frac{1}{A_{s}}, s \leqq t\right)\left(e_{t}\right)^{-\mu}\right] d \eta
\end{aligned}
$$

or, equivalently,

$$
\begin{aligned}
\int_{0}^{\infty} e^{-\eta} E\left[F \left(\frac{1}{A_{s}}+2 \eta, s\right.\right. & \left.\leqq t)\left(\eta e_{t}\right)^{\mu}\right] \frac{d \eta}{\eta} \\
& =\int_{0}^{\infty} e^{-\eta} E\left[F\left(\frac{1}{A_{s}}, s \leqq t\right)\left(\frac{\eta}{e_{t}}\right)^{\mu}\right] \frac{d \eta}{\eta} .
\end{aligned}
$$

Since this equality is true for every $\mu>0$, we also obtain

$$
\begin{aligned}
\int_{0}^{\infty} e^{-\eta} E\left[f ( \eta e _ { t } ) F \left(\frac{1}{A_{s}}\right.\right. & +2 \eta, s \leqq t)] \frac{d \eta}{\eta} \\
& =\int_{0}^{\infty} e^{-\eta} E\left[f\left(\eta / e_{t}\right) F\left(\frac{1}{A_{s}}, s \leqq t\right)\right] \frac{d \eta}{\eta}
\end{aligned}
$$

for every non-negative Borel function $f$ on $\mathbf{R}_{+}$. Therefore, using the Fubini theorem and by obvious changes of variables, we get

$$
\begin{aligned}
\int_{0}^{\infty} E\left[\operatorname { e x p } ( - \eta / e _ { t } ) F \left(\frac{1}{A_{s}}\right.\right. & \left.\left.+2 \eta / e_{t}, s \leqq t\right)\right] f(\eta) \frac{d \eta}{\eta} \\
& =\int_{0}^{\infty} E\left[\exp \left(-\eta e_{t}\right) F\left(\frac{1}{A_{s}}, s \leqq t\right)\right] f(\eta) \frac{d \eta}{\eta},
\end{aligned}
$$

which yields the identity (4.1).

\section{§5. Relationship with the GIG Laws}

5.1. Proof of the identity (1.4). a) We first prove the identity for $\mu=0$. We start again from (4.1) or (4.2). With the help of Proposition 2.1, we see that this identity is in fact true for the (regular) conditional probability distribution given $\mathcal{Z}_{t}, Q_{t}^{\omega, z}=P\left(\cdot \mid \mathcal{Z}_{t}, Z_{t}(\omega)=z\right)$ :

$$
\begin{aligned}
& E^{Q_{t}^{\omega, z}}\left[\exp \left(-\eta e_{t}\right) F\left(B_{s}, s \leqq t\right)\right] \\
& =E^{Q_{t}^{\omega, z}}\left[\exp \left(-\eta / e_{t}\right) F\left(T_{2 \eta / e_{t}}(B)_{s}, s \leqq t\right)\right], \quad \eta>0,
\end{aligned}
$$


where $e_{t}=\exp \left(B_{t}\right)$. In particular, for $F=\varphi\left(1 / A_{t}\right)$, we have

$$
E^{Q_{t}^{\omega, z}}\left[\exp \left(-\eta e_{t}\right) \varphi\left(\frac{1}{z e_{t}}\right)\right]=E^{Q_{t}^{\omega, z}}\left[\exp \left(-\eta / e_{t}\right) \varphi\left(\frac{2 \eta+1 / z}{e_{t}}\right)\right] .
$$

Let us assume (for simplicity) that, under $Q_{t}^{\omega, z}, e_{t}$ admits a density $g_{z}(x)$. Then we deduce from (5.2) that

$$
g_{z}((2 \eta+1 / z) v)(2 \eta+1 / z) \exp \left(-\frac{\eta}{(2 \eta+1 / z) v}\right)=g_{z}(v / z) z^{-1} \exp (-\eta v / z) .
$$

Taking $v=1$ and writing $u=2 \eta+1 / z$, we obtain

$$
g_{z}(u)=C_{z} \frac{1}{u} \exp \left(-\frac{1}{2 z}\left(u+\frac{1}{u}\right)\right), \quad u>1 / z
$$

where

$$
C_{z}=g_{z}(1 / z) z^{-1} \exp \left(\frac{1+z^{-2}}{2}\right)
$$

Next, in (5.3), we consider $\eta, v$ satisfying $(2 \eta+1 / z) v z=1$. Then, setting $u=v / z$ and noting

$$
0<u=\frac{1}{z+2 \eta z^{2}}<\frac{1}{z} \quad \text { and } \quad \eta=\frac{1}{2}\left(\frac{1}{z^{2} u}-\frac{1}{z}\right)
$$

we obtain

$$
\begin{aligned}
g_{z}(u) & =g_{z}(1 / z) z^{-1} \exp (-z \eta) \frac{1}{u} \exp (\eta u) \\
& =C_{z} \frac{1}{u} \exp \left(-\frac{1}{2 z}\left(u+\frac{1}{u}\right)\right), \quad 0<u<1 / z,
\end{aligned}
$$

and (1.4) for $\mu=0$ since $g_{z}(u)$ is a probability density and the normalizing constant $C_{z}$ is, from (5.6) below, equal to $\left(2 K_{0}(1 / z)\right)^{-1}$.

b) We then obtain the general result (1.4) for any $\mu$ by using the CameronMartin theorem. In fact, by using (1.4) for $\mu=0$, we obtain for every nonnegative Borel function $f$ and a non-negative adapted functional $F$

$$
\begin{aligned}
& E\left[f\left(e_{t}^{(\mu)}\right) F\left(Z_{s}^{(\mu)}, s \leqq t\right)\right] \\
= & E\left[E\left[f\left(e_{t}\right) \exp \left(\mu B_{t}\right) \mid \mathcal{Z}_{t}\right] F\left(Z_{s}, s \leqq t\right) e^{-\mu^{2} t / 2}\right] \\
= & e^{-\mu^{2} t / 2} E\left[\frac{1}{2 K_{0}\left(1 / Z_{t}\right)} F\left(Z_{s}, s \leqq t\right) \int_{0}^{\infty} f(x) x^{\mu-1} \exp \left(-\frac{1}{2 Z_{t}}\left(x+\frac{1}{x}\right)\right) d x\right] \\
= & E\left[\frac{\left(e_{t}^{(\mu)}\right)^{-\mu}}{2 K_{0}\left(1 / Z_{t}^{(\mu)}\right)} F\left(Z_{s}^{(\mu)}, s \leqq t\right) \int_{0}^{\infty} f(x) x^{\mu-1} \exp \left(-\frac{1}{2 Z_{t}^{(\mu)}}\left(x+\frac{1}{x}\right)\right) d x\right] .
\end{aligned}
$$


Therefore we obtain

$$
\begin{aligned}
P\left(e_{t}^{(\mu)}\right. & \left.\in d x \mid \mathcal{Z}_{t}^{(\mu)}, Z_{t}^{(\mu)}=z\right) \\
& =\frac{E\left[\left(e_{t}^{(\mu)}\right)^{-\mu} \mid \mathcal{Z}_{t}^{(\mu)}, Z_{t}^{(\mu)}=z\right]}{2 K_{0}(1 / z)} x^{\mu-1} \exp \left(-\frac{1}{2 z}\left(x+\frac{1}{x}\right)\right) d x
\end{aligned}
$$

and the constant term on the right hand side equals $\left(2 K_{\mu}(1 / z)\right)^{-1}$.

5.2. The GIG laws. We check that the absolute continuity relationship (1.6) agrees with our formula (1.4) for the conditional law of $e_{t}^{(\mu)} \equiv \exp \left(B_{t}^{(\mu)}\right)$ given $\mathcal{Z}_{t}^{(\mu)}$. The general result for every $\mu$ follows from the special case of $\mu=0$ as we have seen in the previous section, and we assume $\mu=0$ in this subsection.

It is easily shown that (1.6) entails

$$
E\left[\exp \left(-\frac{\alpha}{2}\left(e_{t}-\frac{1}{(1+\alpha z) e_{t}}\right)\right) \mid \mathcal{Z}_{t}, Z_{t}=z\right]=1 .
$$

We can show that this property is implied by (1.4) as a consequence of the following elementary lemma.

Lemma 5.1. Assume that a random variable $X$ is distributed as in (1.4) with $\xi=1 / z$ and $\mu=0$. Then, for $a, b>0$, in order that

$$
E\left[\exp \left(-\frac{a X}{2}+\frac{b}{2 X}\right)\right]=1
$$

holds, one must have $(a+\xi)(\xi-b)=\xi^{2}$. Consequently, (5.5) holds.

Proof. The lemma follows easily from the identity

$$
K_{0}(m n)=\frac{1}{2} \int_{0}^{\infty} \exp \left(-\frac{1}{2}\left(m^{2} u+\frac{n^{2}}{u}\right)\right) \frac{d u}{u}, \quad m, n>0,
$$

that is, the integral on the right hand side only depends on the product $m n$.

\section{$\S 6$. Proof of Theorem 1.5 and Comparison to Theorem 1.1}

6.1. Proof of Theorem 1.5 in the case $\boldsymbol{\mu}=\mathbf{0}$. From the previous discussions about the transform $T_{\alpha}$ and the associated $\sigma$-fields, it suffices for the proof of (1.9) for $\mu=0$ to consider the functionals of the form

$$
G(\phi(s), s \leqq t)=F\left(Z_{s}(\phi), s \leqq t\right) f(\exp (\phi(t)))
$$


for a generic non-negative functional $F$ and a Borel non-negative function $f$.

Thus, using (6.1) and the fact that $Z \circ T_{\alpha}=Z$, we see that the equality (1.9) for $\mu=0$, with $\Delta_{\alpha}$ in place of $\Delta_{\alpha}\left(e_{t}, \alpha A_{t}\right)$, is equivalent to

$$
E\left[f\left(\frac{e_{t}}{1+\alpha e_{t} Z_{t}}\right) \mid \mathcal{Z}_{t}\right]=E\left[f\left(e_{t}\right) \Delta_{\alpha} \mid \mathcal{Z}_{t}\right]
$$

where $\mathcal{Z}_{t}=\mathcal{Z}_{t}^{(0)}$. As a consequence, it suffices to find a function $\Delta_{\alpha, z}(v)$ such that

$$
E\left[f\left(\frac{e_{t}}{1+\alpha e_{t} Z_{t}}\right) \mid \mathcal{Z}_{t}, Z_{t}=z\right]=E\left[f\left(e_{t}\right) \Delta_{\alpha, z}\left(e_{t}\right) \mid \mathcal{Z}_{t}, Z_{t}=z\right] .
$$

We now use (1.4) for $\mu=0$. Then some elementary computations lead us to the conclusion that the function

$$
\Delta_{\alpha, z}(v)=\frac{\mathbf{1}_{\{\alpha z v<1\}}}{(1-\alpha z v)} \exp \left(-\frac{\alpha}{2}\left(\frac{v^{2}}{1-\alpha z v}-1\right)\right)
$$

satisfies (6.2). The rest of the proof is easy.

6.2. Bessel processes and bridges. The form of the Radon-Nikodym density in (1.9) for $\mu=0$ leads us to relate Theorem 1.5 for $\mu=0$ to the Lamperti (implicit) representation of geometric Brownian motions in terms of the Bessel processes: there exists a Bessel process $R^{(\mu)}=\left\{R_{t}^{(\mu)}, t \geqq 0\right\}$ of dimension $\delta=2(1+\mu)$ or of index $\mu$ starting from 1 such that

$$
\exp \left(B_{t}^{(\mu)}\right)=R_{A_{t}^{(\mu)}}^{(\mu)}, \quad t \geqq 0 .
$$

Indeed, the form of the function $\Delta_{\alpha}(v, u)$ in the statement of Theorem 1.5 for $\mu=0$ invites to relate this quantity to the martingale

$$
D_{u}^{\alpha}=\frac{1}{1-\alpha u} \exp \left(-\frac{\alpha}{2}\left(\frac{R_{u}^{2}}{1-\alpha u}-1\right)\right), \quad u<1 / \alpha,
$$

where $R=R^{(0)}$ is a two-dimensional Bessel process starting from 1 .

We now recall the well known Laplace transform formula for the distribution of the squared Bessel process, which will imply the martingale property of $\left\{D_{u}^{\alpha}, 0 \leqq u<1 / \alpha\right\}$ : letting $X^{x}=\left\{X_{t}^{x}, t \geqq 0\right\}$ be a $\delta$-dimensional squared Bessel process starting from $x$, one has

$$
E\left[\exp \left(-\lambda X_{t}^{x}\right)\right]=(1+2 \lambda t)^{-\delta / 2} \exp \left(-\frac{\lambda x}{1+2 \lambda t}\right)
$$

for $\lambda>0$ (see [18], p.441). Then we can characterize the martingales of the form (6.4) for the squared Bessel processes. 
Lemma 6.1. Let $T>0$ be fixed and $h, k:[0, T) \rightarrow \mathbf{R}_{+}$be two deterministic functions such that

$$
M_{u}^{h, k}=k(u) \exp \left(-h(u) X_{u}\right), \quad u<T,
$$

is a martingale with respect to the law $Q_{x}^{\delta}$ of $X^{x}$ and $\mathcal{R}_{u} \equiv \sigma\left\{X_{s}, s \leqq u\right\}$. Then there exist positive constants $C$ and $K$ such that

$$
h(u)=\frac{1}{2(C-u)} \quad \text { and } \quad k(u)=K(C-u)^{-\delta / 2},
$$

so that $\left\{M_{u}^{h, k}\right\}$ is defined on $[0, C)$ (i.e., the optimal value of $T$ is $C$ ). Moreover it holds that

$$
E^{Q_{x}^{\delta}}\left[M_{u}^{h, k}\right]=K C^{-\delta / 2} \exp \left(-\frac{x}{2 C}\right)
$$

Proof. By formula (6.5), we have

$$
\begin{aligned}
E^{Q_{x}^{\delta}} & {\left[k(u) \exp \left(-h(u) X_{u}\right) \mid \mathcal{R}_{s}\right] } \\
& =\frac{k(u)}{(1+2 h(u)(u-s))^{\delta / 2}} \exp \left(-\frac{h(u) X_{s}}{1+2 h(u)(u-s)}\right)
\end{aligned}
$$

for $s<u<T$ and the assumption implies

$$
k(s)=\frac{k(u)}{(1+2 k(u)(u-s))^{\delta / 2}} \quad \text { and } \quad h(s)=\frac{h(u)}{1+2 h(u)(u-s)} .
$$

In particular, we have

$$
\frac{1}{h(s)}+2 s=\frac{1}{h(u)}+2 u,
$$

that is, the function $(h(u))^{-1}+2 u, u<T$, is constant. Therefore there exists a constant $C>0$ such that

$$
h(u)=\frac{1}{2(C-u)} .
$$

The rest of the proof is easy and is omitted.

We now fix $x>0$ and $T>0$. Noting that the squared Bessel process $X=\left\{X_{u}^{x}\right\}$ of dimension $\delta$ is given as the solution of the stochastic differential equation

$$
d X_{u}=2 \sqrt{X_{u}} d w_{u}+\delta d u ; \quad X_{0}=x,
$$

for a standard Brownian motion $\left\{w_{u}, u \geqq 0\right\}$ and that we have

$$
\frac{X_{u}}{T-u}-\frac{x}{T}=\int_{0}^{u} \frac{2 \sqrt{X_{s}}}{T-s} d w_{s}+\int_{0}^{u} \frac{X_{s}}{(T-s)^{2}} d s+\delta \log \left(\frac{T}{T-u}\right), \quad u<T,
$$


we consider the martingale probability density

$$
\Delta_{u}^{T}=(1-u / T)^{-\delta / 2} \exp \left(-\frac{1}{2}\left(\frac{X_{u}}{T-u}-\frac{x}{T}\right)\right), \quad u<T,
$$

with respect to $\left(Q_{x}^{\delta}, \mathcal{R}_{u}\right)$. Then, recalling that the squared Bessel bridge $X^{\delta, x, T}=\left\{X_{u}^{\delta, x, T}, u \leqq T\right\}$ of dimension $\delta$ which starts from $x$ at time 0 and ends at 0 at time $T$ is given as the solution of the stochastic differential equation

$$
d X_{u}=2 \sqrt{X_{u}} d w_{u}+\delta d u-\frac{2 X_{u}}{T-u} d u
$$

(cf. [18], p.468), we can show, as a consequence of a simple application of the Maruyama-Girsanov theorem, that, if we consider the probability measure $Q_{x}^{\delta, T}$ given by

$$
\left.Q_{x}^{\delta, T}\right|_{\mathcal{R}_{u}}=\left.\Delta_{u}^{T} \cdot Q_{x}^{\delta}\right|_{\mathcal{R}_{u}}, \quad u<T,
$$

then $Q_{x}^{\delta, T}$ gives rise to the distribution of the squared Bessel bridge.

6.3. Proof of Theorem $\mathbf{1 . 5}$ for general $\boldsymbol{\mu} \geqq \mathbf{0}$. Formula (1.9) is proved in the same way as we did in Subsection 6.1. Then, we deduce (1.10) from it because, letting $\tau_{u}^{(\mu)}$ be the inverse function of $A_{t}^{(\mu)}$ and using (6.3), we have

$$
\Delta_{\alpha}^{(\mu)}\left(e_{\tau_{u}^{(\mu)}}^{(\mu)}, \alpha A_{\tau_{u}^{(\mu)}}^{(\mu)}\right)=\frac{1_{\{\alpha u<1\}}}{(1-\alpha u)^{\mu+1}} \exp \left(-\frac{1}{2}\left(\frac{\left(R_{u}^{(\mu)}\right)^{2}}{\alpha^{-1}-u}-\frac{1}{\alpha^{-1}}\right)\right) .
$$

6.4. Comparison of Theorems 1.1 and 1.5. We now present a further effort to synthesize the contents of both Theorems 1.1 and 1.5 and to compare them. We set

$$
g_{z}^{(\mu)}(x)=\frac{1}{2 K_{\mu}(1 / z)} x^{\mu-1} \exp \left(-\frac{1}{2 z}\left(x+\frac{1}{x}\right)\right)
$$

(in agreement with our notation in (5.4) for $\mu=0$ ), which is the density of the GIG law appearing on the right hand side of (1.4).

Proposition 6.2. (i) For all $x>0$ satisfying $\alpha z x<1$, one has

$$
\frac{1}{(1-\alpha z x)^{2}} g_{z}^{(\mu)}\left(\frac{x}{1-\alpha z x}\right)=g_{z}^{(\mu)}(x) \Delta_{\alpha}^{(\mu)}(x, \alpha z x)
$$

and

$$
\begin{aligned}
E\left[G \left(T_{\alpha}\left(B^{(\mu)}\right)_{s}, s\right.\right. & \left.\leqq t) \mid e_{t}^{(\mu)}=\frac{x}{1-\alpha z x}, Z_{t}^{(\mu)}=z\right] \\
& =E\left[G\left(B_{s}^{(\mu)}, s \leqq t\right) \mid e_{t}^{(\mu)}=x, Z_{t}^{(\mu)}=z\right] .
\end{aligned}
$$


(ii) For every $x, z>0$, one has

$$
(1+\alpha z) g_{z}^{(\mu)}((1+\alpha z) x)=g_{z}^{(\mu)}(x) \Gamma_{\alpha}^{(\mu)}(x, z)
$$

and

$$
\begin{aligned}
E\left[G \left(T_{\left.\alpha / e_{t}^{(\mu)}\left(B^{(\mu)}\right)_{s}, s \leqq t\right) \mid e_{t}^{(\mu)}}\right.\right. & \left.=(1+\alpha z) x, Z_{t}^{(\mu)}=z\right] \\
& =E\left[G\left(B_{s}^{(\mu)}, s \leqq t\right) \mid e_{t}^{(\mu)}=x, Z_{t}^{(\mu)}=z\right]
\end{aligned}
$$

Proof. Although one might argue that the whole contents of the above identities may essentially be found in our proofs of Theorems 1.5 and 1.1, we find it convenient to give a few details for, say, (6.6) and (6.7).

From formula (1.9), we deduce

$$
\begin{aligned}
E\left[G \left(T_{\alpha}\left(B^{(\mu)}\right)_{s}, s\right.\right. & \left.\leqq t) f\left(Z_{t}^{(\mu)}\right) h\left(\frac{e_{t}^{(\mu)}}{1+\alpha A_{t}^{(\mu)}}\right)\right] \\
& =E\left[G\left(B_{s}^{(\mu)}, s \leqq t\right) f\left(Z_{t}^{(\mu)}\right) h\left(e_{t}^{(\mu)}\right) \Delta_{\alpha}^{(\mu)}\left(e_{t}^{(\mu)}, \alpha A_{t}^{(\mu)}\right)\right]
\end{aligned}
$$

for every non-negative adapted functional $G$ and for all non-negative measurable functions $f$ and $h$. Then, writing both hand sides of (6.10) as the integrals with respect to the joint law of $\left(e_{t}^{(\mu)}, Z_{t}^{(\mu)}\right)$ and using (1.4), we obtain (6.6) and (6.7) after routine manipulations.

We now compare the identities (6.7) and (6.9). For this purpose we remark that the left hand side of (6.9) may be written as

$$
E\left[G\left(T_{\frac{\alpha}{(1+\alpha z) x}}\left(B^{(\mu)}\right)_{s}, s \leqq t\right) \mid e_{t}^{(\mu)}=(1+\alpha z) x, Z_{t}^{(\mu)}=z\right] .
$$

Then, making the change of variables: $\beta=\alpha((1+\alpha z) x)^{-1}$, we obtain from (6.9)

$$
\begin{aligned}
E\left[G \left(T_{\beta}\left(B^{(\mu)}\right)_{s}, s\right.\right. & \left.\leqq t) \mid e_{t}^{(\mu)}=\frac{x}{1-\beta z x}, Z_{t}^{(\mu)}=z\right] \\
& =E\left[G\left(B_{s}^{(\mu)}, s \leqq t\right) \mid e_{t}^{(\mu)}=x, Z_{t}^{(\mu)}=z\right]
\end{aligned}
$$

for $\beta z x<1$. This is nothing else but formula (6.7).

6.5. Adding an independent "drift" to $\left\{1 / A_{t}^{(\mu)}, t>0\right\}$. We show that, if $X$ is a non-negative random variable independent of $B^{(\mu)}$, then, for any $t>0$, the probability law of $\left\{\left(A_{s}^{(\mu)}\right)^{-1}+X, s \leqq t\right\}$ is absolutely continuous with respect to that of $\left\{\left(A_{s}^{(-\mu)}\right)^{-1}, s \leqq t\right\}$, and hence with respect to that of the same quantity for Brownian motion. 
Proposition 6.3. Let $\mu \geqq 0$ and let $X$ be a non-negative random variable, independent of $B^{(\mu)}$, with density $p(x)$ which is positive a.e. Then, for any non-negative adapted functional $F$, one has

$$
E\left[F\left(\frac{1}{A_{s}^{(\mu)}}+X, s \leqq t\right)\right]=E\left[F\left(\frac{1}{A_{s}^{(-\mu)}}, s \leqq t\right) \Lambda_{\mu}\left(A_{t}^{(-\mu)},\left(e_{t}^{(\mu)}\right)^{2}\right)\right]
$$

where, setting $h_{\mu}(x)=x^{\mu-1} e^{-x / 2}$ and $q_{\mu}(x)=p(x) / h_{\mu}(x)$,

$$
\Lambda_{\mu}(a, y)=2^{\mu} \int_{0}^{\infty} k^{\mu-1} e^{-k / 2} q_{\mu}\left(\frac{1}{a+y / 2 k}\right) d k .
$$

Proof. At first we assume $\mu>0$ and use (1.1). One has

$$
\begin{aligned}
E\left[F\left(\frac{1}{A_{s}^{(\mu)}}+X, s \leqq t\right)\right] & =\int_{0}^{\infty} E\left[F\left(\frac{1}{A_{s}^{(\mu)}}+x, s \leqq t\right)\right] p(x) d x \\
& =\int_{0}^{\infty} \tilde{q}_{\mu}(x) E\left[F\left(\frac{1}{A_{s}^{(\mu)}}+x, s \leqq t\right)\right] \tilde{h}_{\mu}(x) d x \\
& =E\left[f\left(\frac{1}{A_{s}^{(\mu)}}+\frac{1}{\tilde{A}_{\infty}^{(-\mu)}}, s \leqq t\right) \tilde{q}_{\mu}\left(\frac{1}{\tilde{A}_{\infty}^{(-\mu)}}\right)\right]
\end{aligned}
$$

where $\tilde{h}_{\mu}(x)=\left(2^{\mu} \Gamma(\mu)\right)^{-1} x^{\mu-1} e^{-x / 2}$ is the density of $2 \gamma_{\mu}$ and $\tilde{q}_{\mu}(x)=$ $p(x) / \tilde{h}_{\mu}(x)$. Since identity (1.1) implies

$$
\left(\left\{\frac{1}{A_{t}^{(-\mu)}}, t>0\right\}, \frac{1}{A_{\infty}^{(-\mu)}}\right) \stackrel{(\text { law })}{=}\left(\left\{\frac{1}{A_{t}^{(\mu)}}+\frac{1}{\tilde{A}_{\infty}^{(-\mu)}}, t>0\right\}, \frac{1}{\tilde{A}_{\infty}^{(-\mu)}}\right),
$$

the expectation (6.12) is equal to

$$
E\left[F\left(\frac{1}{A_{s}^{(-\mu)}}, s \leqq t\right) \tilde{q}_{\mu}\left(\frac{1}{A_{\infty}^{(-\mu)}}\right)\right]
$$

It then remains to compute $E\left[\tilde{q}_{\mu}\left(\left(A_{\infty}^{(-\mu)}\right)^{-1}\right) \mid \mathcal{B}_{t}\right]$, where $\mathcal{B}_{t}=\sigma\left\{B_{s}^{(-\mu)}, s \leqq\right.$ $t\}$. For this we note

$$
A_{\infty}^{(-\mu)}=A_{t}^{(-\mu)}+\int_{t}^{\infty}\left(e_{s}^{(-\mu)}\right)^{2} d s=A_{t}^{(-\mu)}+\left(e_{t}^{(-\mu)}\right)^{2} \tilde{A}_{\infty}^{(-\mu)},
$$


where $\tilde{A}_{\infty}^{(-\mu)}$ is a copy of $A_{\infty}^{(-\mu)}$, independent of $\mathcal{B}_{t}$. Then one obtains

$$
\begin{aligned}
E\left[\tilde{q}_{\mu}\left(\frac{1}{\tilde{A}_{\infty}^{(-\mu)}}\right) \mid \mathcal{B}_{t}\right] & =E\left[\tilde{q}_{\mu}\left(\frac{1}{A_{t}^{(-\mu)}+\left(e_{t}^{(-\mu)}\right)^{2} \tilde{A}_{\infty}^{(-\mu)}}\right) \mid \mathcal{B}_{t}\right] \\
& =\int_{0}^{\infty} \tilde{q}_{\mu}\left(\frac{1}{A_{t}^{(-\mu)}+\left(e_{t}^{(-\mu)}\right)^{2} / 2 k}\right) \frac{1}{\Gamma(\mu)} k^{\mu-1} e^{-k} d k \\
& =\int_{0}^{\infty} 2^{\mu} q_{\mu}\left(\frac{1}{A_{t}^{(-\mu)}+\left(e_{t}^{(-\mu)}\right)^{2} / 2 k}\right) k^{\mu-1} e^{-k} d k
\end{aligned}
$$

and the result (6.11) for $\mu>0$.

To deal with the case $\mu=0$, it suffices to let $\mu \downarrow 0$ in formula (6.11) for $\mu>0$.

Remark 6.1. Clearly, it is possible to unify the result in the independent case given in the preceding proposition with that of Theorem 1.1 under the more general condition that the conditional law of $X$ given $\mathcal{Z}_{t}^{(\mu)}$ is equivalent to the Lebesgue measure. We leave the details for the interested reader.

\section{$\S 7 . \quad$ Pseudo Martingale Properties of the Densities $\Gamma_{\alpha}^{(\mu)}\left(e_{t}^{(\mu)}, Z_{t}^{(\mu)}\right)$}

7.1. $\left(\mathcal{Z}_{t}^{(\mu)}\right)$-anticipative martingales. Since the left hand side of (1.6) involves $e_{t}^{(\mu)}$, the stochastic process $\left\{\Gamma_{\alpha}^{(\mu)}\left(e_{t}^{(\mu)}, Z_{t}^{(\mu)}\right), t \geqq 0\right\}$ is not a $\left(\mathcal{B}_{t}\right)$ martingale (see Subsection 7.2 below for a more precise discussion). Nonetheless, as we discussed in Subsection 3.2, there is the relation

$$
E\left[\Gamma_{\alpha}^{(\mu)}\left(e_{t}^{(\mu)}, Z_{t}^{(\mu)}\right) \mid \mathcal{Z}_{t}^{(\mu)}\right]=1 .
$$

More generally, we would like to introduce the notion of a $\left(\mathcal{Z}_{t}^{(\mu)}\right)$-anticipative martingale as a stochastic process $\left\{M_{t}, t \geqq 0\right\}$ such that $E\left[M_{t} \mid \mathcal{Z}_{t}^{(\mu)}\right]$ is a $\left(\mathcal{Z}_{t}^{(\mu)}\right)$ martingale. Thus, in particular, we claim that $\left\{\Gamma_{\alpha}^{(\mu)}\left(e_{t}^{(\mu)}, Z_{t}^{(\mu)}\right), t \geqq 0\right\}$ is a $\left(\mathcal{Z}_{t}^{(\mu)}\right)$-anticipative martingale, but is not a $\left(\mathcal{B}_{t}\right)$-martingale. In fact, by using Itô's formula, we can write

$$
\Gamma_{\alpha}^{(\mu)}\left(e_{t}^{(\mu)}, Z_{t}^{(\mu)}\right)-\int_{0}^{t} \gamma_{\alpha}^{(\mu)}\left(e_{s}^{(\mu)}, Z_{s}^{(\mu)}\right) d s
$$

is a $\left(\mathcal{B}_{t}\right)$-martingale for some function $\gamma_{\alpha}^{(\mu)}$, which, as a consequence of (7.1), is equivalent to

$$
E\left[\gamma_{\alpha}^{(\mu)}\left(e_{s}^{(\mu)}, Z_{s}^{(\mu)}\right) \mid \mathcal{Z}_{s}^{(\mu)}\right]=0
$$


or, with the help of (1.4),

$$
\int_{0}^{\infty} \gamma_{\alpha}^{(\mu)}(x, z) x^{\mu-1} \exp \left(-\frac{1}{2 z}\left(x+\frac{1}{x}\right)\right) d x=0, \quad z>0,
$$

and these identities provide us with further $\left(\mathcal{Z}_{t}^{(\mu)}\right)$-anticipative martingales.

The above discussion is a very particular case of the following well known fact (see, e.g., [10]).

Lemma 7.1. Let $\mathcal{G}_{t} \subseteq \mathcal{F}_{t}, t \geqq 0$, be two filtrations and $\left\{X_{t}, t \geqq 0\right\}$ and $\left\{x_{t}, t \geqq 0\right\}$ be two $\left(\mathcal{F}_{t}\right)$-adapted measurable stochastic processes such that $\left\{X_{t}-\int_{0}^{t} x_{s} d s\right\}$ is an $\left(\mathcal{F}_{t}\right)$-martingale. Then

$$
E\left[X_{t} \mid \mathcal{G}_{t}\right]-\int_{0}^{t} E\left[x_{s} \mid \mathcal{G}_{s}\right] d s, \quad t \geqq 0,
$$

is a $\left(\mathcal{G}_{t}\right)$-martingale. In particular, if $E\left[X_{t} \mid \mathcal{G}_{t}\right]$ does not depend on $t$ and if $\left\{E\left[x_{s} \mid \mathcal{G}_{s}\right], s \geqq 0\right\}$ admits a continuous version, then this version is identically equal to 0 .

Proof. For $s<t$ and $G_{s} \in \mathcal{G}_{s}$, we have

$$
E\left[\mathbf{1}_{G_{s}}\left(X_{t}-X_{s}-\int_{s}^{t} x_{u} d u\right)\right]=0 .
$$

On the other hand, this expectation is equal to

$$
E\left[\mathbf{1}_{G_{s}}\left(E\left[X_{t} \mid \mathcal{G}_{t}\right]-E\left[X_{s} \mid \mathcal{G}_{s}\right]-\int_{s}^{t} E\left[x_{u} \mid \mathcal{G}_{u}\right] d u\right)\right] .
$$

Hence we obtain the result.

The following remarks may also be useful.

Proposition 7.2. (i) Assume that, for a function $f: \mathbf{R} \times \mathbf{R}_{+} \rightarrow \mathbf{R}$, $\left\{f\left(B_{t}^{(\mu)}, Z_{t}^{(\mu)}\right), t \geqq 0\right\}$ is an anticipative $\left(\mathcal{Z}_{t}^{(\mu)}\right)$-martingale. Then, one has $E\left[f\left(B_{t}^{(\mu)}, Z_{t}^{(\mu)}\right) \mid \mathcal{Z}_{t}^{(\mu)}\right]=f(0,0)$.

(ii) Assume $\mu=0$. Then, if a function $\varphi: \mathbf{R}_{+} \times \mathbf{R}_{+} \rightarrow \mathbf{R}$ satisfies $\varphi(x, z)=$ $-\varphi(1 / x, z)$, one has $E\left[\varphi\left(e_{t}, Z_{t}\right) \mid \mathcal{Z}_{t}\right]=0$.

Proof. (i) From formula (1.4), we know that there exists a function $\tilde{f}$ : $\mathbf{R} \rightarrow \mathbf{R}$ (which we could write explicitly in terms of $f$ ) such that

$$
E\left[f\left(B_{t}^{(\mu)}, Z_{t}^{(\mu)}\right) \mid \mathcal{Z}_{t}^{(\mu)}\right]=\tilde{f}\left(Z_{t}^{(\mu)}\right) .
$$


But, the only functions such that $\left\{\varphi\left(Z_{t}^{(\mu)}\right), t \geqq 0\right\}$ is a $\left(\mathcal{Z}_{t}^{(\mu)}\right)$-martingale are constant functions. Indeed, $\varphi$ is then an affine transform of a scale function $S$ for $\left\{Z_{t}^{(\mu)}\right\}$ and $\left\{S\left(Z_{t}^{(\mu)}\right), t \geqq 0\right\}$ is not a martingale.

(ii) This follows from the fact that the conditional law of $e_{t} \equiv \exp \left(B_{t}\right)$ given $\mathcal{Z}_{t}$ is invariant under the mapping $x \rightarrow 1 / x$.

7.2. Loss of martingale property of the density. We now discuss precisely the loss of martingale property for $\left\{\Gamma_{\alpha}^{(\mu)}\left(e_{t}^{(\mu)}, Z_{t}^{(\mu)}\right), t \geqq 0\right\}$, which in fact is replaced by an interesting interplay between the respective infinitesimal generators $\mathcal{L}^{(\mu)}$ and $L^{(\mu)}$ of the two-dimensional diffusion process $\left\{\left(B_{t}^{(\mu)}, Z_{t}^{(\mu)}\right), t \geqq\right.$ $0\}$ and of the one-dimensional Brownian motion $B^{(\mu)}=\left\{B_{t}^{(\mu)}\right\}$ with drift $\mu$ :

$$
\mathcal{L}^{(\mu)}=\frac{1}{2} \frac{\partial^{2}}{\partial b^{2}}+\frac{1}{2} z^{2} \frac{\partial^{2}}{\partial z^{2}}-z \frac{\partial^{2}}{\partial b \partial z}+\mu \frac{\partial}{\partial b}+\left\{\left(\frac{1}{2}-\mu\right) z+e^{b}\right\} \frac{\partial}{\partial z},
$$

and

$$
L^{(\mu)}=\frac{1}{2} \frac{d^{2}}{d \beta^{2}}+\mu \frac{d}{d \beta} .
$$

In order to proceed further, we need to introduce a few notations. If $F_{\beta}(u, v)$ is a function of three real arguments, we shall write $\left(P_{t}^{(\mu)} F\right)_{\beta}(u, v)$ for $P_{t}^{(\mu)}\left(F_{.}(u, v)\right)(\beta)$, where $\left\{P_{t}^{(\mu)}\right\}$ is the semigroup of $B^{(\mu)}$. We also write $\hat{\Gamma}_{y}^{(\mu)}(u, v)$ for $\Gamma_{\exp (-y)}^{(\mu)}(u, v)$.

We may now state the following.

Theorem 7.3. Denote by $\left\{Q_{t}^{(\mu)}\right\}$ and $\left\{P_{t}^{(\mu)}\right\}$ the respective semigroups of $\left\{\left(B_{t}^{(\mu)}, Z_{t}^{(\mu)}\right)\right\}$ and $B^{(\mu)}$. Moreover let $\mathcal{L}^{(\mu)}$ and $L^{(\mu)}$ be the restrictions of their infinitesimal generators to $C^{2}$ functions. Then the following identities are satisfied:

$$
\left(Q_{t}^{(\mu)} \hat{\Gamma}_{\beta}^{(\mu)}\right)(b, z)=\left(P_{t}^{(\mu)} \hat{\Gamma}^{(\mu)}\right)_{\beta}(b, z)
$$

and, consequently,

$$
\mathcal{L}^{(\mu)} \hat{\Gamma}_{\beta}^{(\mu)}(b, z)=\left(L^{(\mu)} \hat{\Gamma}^{(\mu)}\right)_{\beta}(b, z)
$$

Proof. We start from writing formula (1.6) for two times $s$ and $t, s \leqq t$, as follows:

$$
\begin{aligned}
& E\left[F\left(B_{u}^{(\mu)}-\log \left(1+\alpha\left(e_{t}^{(\mu)}\right)^{-1} A_{u}^{(\mu)}\right), u \leqq s\right)\right] \\
& \quad=E\left[F\left(B_{u}^{(\mu)}, u \leqq s\right) \Gamma_{\alpha}^{(\mu)}\left(e_{t}^{(\mu)}, Z_{t}^{(\mu)}\right)\right] .
\end{aligned}
$$


Since, on the left hand side, we can write

$$
1+\alpha\left(e_{t}^{(\mu)}\right)^{-1} A_{u}^{(\mu)}=1+\frac{\alpha}{\exp \left(B_{t}^{(\mu)}-B_{s}^{(\mu)}\right)}\left(e_{s}^{(\mu)}\right)^{-1} A_{u}^{(\mu)},
$$

and $B_{t}^{(\mu)}-B_{s}^{(\mu)}$ is independent of $\left\{B_{u}^{(\mu)}, u \leqq s\right\}$, we obtain that the left hand side of (7.4) is, thanks to (1.6) again, equal to

$$
E\left[F\left(B_{u}^{(\mu)}, u \leqq s\right) \Gamma_{\alpha \exp \left(-\left(B_{t}^{(\mu)}-B_{s}^{(\mu)}\right)\right)}^{(\mu)}\left(e_{s}^{(\mu)}, Z_{s}^{(\mu)}\right)\right],
$$

so that, comparing the right hand side of (7.4) and (7.5), we obtain (7.2). Formula (7.3) follows from (7.2).

It may be worth while presenting the discussion of the preceeding theorem in the following general framework.

Proposition 7.4. Let $\left(\mathcal{F}_{t}, t \geqq 0\right)$ be a filtration and, with respect to it, let $\left\{\xi_{t}, t \geqq 0\right\}$ be a Lévy process with semigroup $\left\{P_{t}\right\}$ and let $\left\{Y_{t}, t \geqq 0\right\}$ be a Markov process with semigroup $\left\{Q_{t}\right\}$, respectively. Assume that there exists a family of transforms $\left\{S_{x}, x \in \mathbf{R}\right\}$ and a function $\Gamma_{x}(y)$ of two variables such that

$$
E\left[F\left(S_{x+\xi_{t}}(Y)_{s}, s \leqq t\right)\right]=E\left[F\left(Y_{s}, s \leqq t\right) \Gamma_{x}\left(Y_{t}\right)\right]
$$

holds for every non-negative adapted functional $F$. Then it holds that

$$
Q_{t}\left(\Gamma_{x}\right)(y)=P_{t}\left(\Gamma^{y}\right)_{x}
$$

where $\Gamma^{y}=\Gamma .(y)$.

\section{§8. Formula (1.6) as an Anticipative Change of Probability Measures}

For simplicity, we consider formula (1.6) for $t=1$ and $\mu=0$. We also find it convenient to use the same notations as those in [4]. For example, we denote a Brownian motion by $\{w(t), t \leqq 1\}$ instead of $\left\{B_{t}, t \leqq 1\right\}$ and also denote by $\left\{e_{t}(w)\right\},\left\{A_{t}(w)\right\},\left\{Z_{t}(w)\right\}$ and so on the corresponding stochastic processes considered so far. Hence, using these notations, we show that the stochastic process $\xi$ given by

$$
\xi_{t}(w)=T_{\alpha / e_{1}(w)}(w)_{t}, \quad t \leqq 1,
$$

solves a stochastic differential equation of the form (1.11) and we also identify the drift $k_{s}(\xi, y)$, as well as $\left\{\eta_{t}(w), t \leqq 1\right\}$, the inverse transform of $\xi$. 
Proposition 8.1. (i) The stochastic process $\xi=\left\{\xi_{t}, t \leqq 1\right\}$ defined by (8.1) is a solution of

$$
d \xi_{t}=d w(t)+k_{t}(\xi(w), w(1)) d t
$$

where

$$
k_{t}(\xi, y)=-\frac{\alpha \exp \left(2 \xi_{t}\right)}{e^{y}-\alpha A_{t}(\xi)}
$$

(ii) It holds that

$$
\eta_{t}(w)=w(t)-\log \left(1-\alpha e^{-\eta_{1}(w)} A_{t}(w)\right)
$$

and

$$
\eta_{1}(w)=w(1)+\log \left(1+\alpha Z_{1}(w)\right)
$$

(iii) It holds that

$$
\int_{0}^{1} k_{t}^{\prime}\left(w, \eta_{1}(w)\right) d t=\alpha Z_{1}(w),
$$

where $k_{t}^{\prime}(w, y)=\frac{d}{d y} k_{t}(w, y)$.

(iv) It holds that

$$
\begin{aligned}
\exp \left(\int_{0}^{1} k_{s}\left(w, \eta_{1}(w)\right) d w(s)-\frac{1}{2} \int_{0}^{1} k_{s}\left(w, \eta_{1}(w)\right)^{2} d s\right) \\
=\left(1+\alpha Z_{1}(w)\right) \exp \left(-\frac{\alpha}{2}\left(e_{1}(w)-\frac{1}{e_{1}(w)+\alpha A_{1}(w)}\right)\right)
\end{aligned}
$$

Remark 8.1. For the stochastic integral on the left hand side of (8.7), see the explanation given on page 324, [4].

The following corollary follows immediately from formula (1.13), using the proposition.

Corollary 8.2. In the particular case where $\xi$ solves the equation (1.13) with the drift $k$ given by (8.3), formula (1.13) becomes

$$
\frac{d P_{\xi}}{d P}=\exp \left(-\frac{\alpha}{2}\left(e_{1}(w)-\frac{1}{e_{1}(w)+\alpha A_{1}(w)}\right)\right),
$$

that is, precisely formula (1.6). 
Proof of Proposition 8.1. (i) We set $\beta=\alpha / e_{1}(w)$ for simplicity. Since we have $\xi_{t}=T_{\beta}(w)_{t}$, it follows from Proposition 2.1 (see also Remark 2.1) that

$$
w(t)=T_{-\beta}(\xi)_{t}=\xi_{t}-\log \left(1-\beta A_{t}(\xi)\right) .
$$

Hence we obtain

$$
\begin{aligned}
\xi_{t} & =w(t)+\log \left(1-\beta A_{t}(\xi)\right)=w(t)-\beta \int_{0}^{t} \frac{\exp \left(2 \xi_{s}\right)}{1-\beta A_{s}(\xi)} d s \\
& =w(t)-\alpha \int_{0}^{t} \frac{\exp \left(2 \xi_{s}\right)}{e_{1}(w)-\alpha A_{s}(\xi)} d s
\end{aligned}
$$

Thus we obtain (8.2).

(ii) Identity (8.8) above yields (8.4). Using again the facts that $\xi_{1}(w)=w(1)-$ $\log \left(1+\alpha Z_{1}(w)\right)$ and that $Z_{t}(w)=Z_{t}(\xi), t \leqq 1$, we obtain

$$
w(1)=\xi_{1}(w)+\log \left(1+\alpha Z_{1}(\xi)\right)
$$

and, therefore, formula (8.5).

(iii) From formula (8.3), we obtain

$$
k_{t}^{\prime}(w, y)=\frac{\alpha e^{y}\left(e_{t}(w)\right)^{2}}{\left(e^{y}-\alpha A_{t}(w)\right)^{2}}=\frac{d}{d t}\left(\frac{e^{y}}{e^{y}-\alpha A_{t}(w)}\right) .
$$

Consequently, we get

$$
\int_{0}^{1} k_{t}^{\prime}(w, y) d t=\frac{e^{y}}{e^{y}-\alpha A_{1}(w)}-1 .
$$

Then, replacing $e^{y}$ by $\exp \left(\eta_{1}(w)\right)=e_{1}(w)+\alpha A_{1}(w)$, we obtain (8.6).

(iv) Let us denote

$$
\begin{aligned}
\theta_{t}(w) & =\exp \left(\int_{0}^{t} k_{s}\left(w, \eta_{1}(w)\right) d w(s)-\frac{1}{2} \int_{0}^{t} k_{s}\left(w, \eta_{1}(w)\right)^{2} d s\right) \\
& \equiv \exp \left(M_{t}-\frac{1}{2}\langle M\rangle_{t}\right)
\end{aligned}
$$

with obvious notation. Moreover we set $m=e_{1}(w)+\alpha A_{1}(w)$ for simplicity. Then, since we have from formulae (8.3) and (8.5)

$$
k_{s}\left(w, \eta_{1}(w)\right)=-\frac{\alpha\left(e_{s}(w)\right)^{2}}{m-\alpha A_{s}(w)}
$$

we obtain

$$
M_{t}=-\alpha \int_{0}^{t} \frac{\alpha\left(e_{s}(w)\right)^{2}}{m-\alpha A_{s}(w)} d w(s)
$$


Noting that $\left\{w_{t}, t \leqq 1\right\}$ is a semimartingale with respect to the enlarged filtration obtained by adding $\sigma\left\{w(1), A_{1}(w)\right\}$ to the original filtration ([4]) and using Itô's formula to develop $-(\alpha / 2)\left(m-\alpha A_{t}(w)\right)^{-1}\left(e_{t}(w)\right)^{2}$, we obtain

$$
\theta_{t}=\frac{1}{1-\alpha A_{t}(w) / m} \exp \left(-\frac{\alpha}{2}\left(\frac{\left(e_{t}(w)\right)^{2}}{m-\alpha A_{t}(w)}-\frac{1}{m}\right)\right) .
$$

Finally, taking $t=1$ in this expression and recalling the definition of $m$, we arrive at

$$
\theta_{1}=\left(1+\alpha Z_{1}(w)\right) \exp \left(-\frac{\alpha}{2}\left(e_{1}(w)-\frac{1}{e_{1}(w)+\alpha A_{1}(w)}\right)\right),
$$

which is precisely formula (8.7).

Now we go back to the Ramer-Kusuoka formula (1.12). For details about the anticipating stochastic calculus, we refer to Nualart [15]. Recall the relation between the Skorohod integral $\delta(H)$ and the generalized Stratonovich integral:

$$
\delta(H)=\int_{0}^{1} H_{s} \circ d w(s)-\frac{1}{2} \int_{0}^{1}\left(\left(D^{+} H\right)_{s}+\left(D^{-} H\right)_{s}\right) d s,
$$

where $H_{s}=k_{s}\left(w, \eta_{1}(w)\right)$,

$$
\left(D^{+} H\right)_{s}=\lim _{u \downarrow s} D_{s} H_{u} \quad \text { and } \quad\left(D^{-} H\right)_{s}=\lim _{u \uparrow s} D_{s} H_{u} .
$$

Moreover the Stratonovich integral on the right hand side is given by

$$
\int_{0}^{1} H_{s} \circ d w(s)=\left.\int_{0}^{1} k_{s}(w, y) \circ d w(s)\right|_{y=\eta_{1}(w)}
$$

in terms of the usual Stratonovich integral. Therefore, using Itô's formula, we obtain

$$
\begin{aligned}
\exp & \left(\int_{0}^{1} H_{s} \circ d w(s)-\frac{1}{2} \int_{0}^{1} H_{s}^{2} d s\right) \\
& =\exp \left(-\frac{\alpha}{2}\left(e_{1}(w)-\frac{1}{\left(1+\alpha Z_{1}(w)\right) e_{1}(w)}\right)\right) \\
& =\Gamma_{\alpha}\left(e_{1}(w), Z_{1}(w)\right),
\end{aligned}
$$

which implies, by the identification of (1.6) and (1.12),

$$
\left|d_{c}(D H)\right|=\exp \left(\frac{1}{2} \int_{0}^{1}\left(\left(D^{+} H\right)_{s}+\left(D^{-} H\right)_{s}\right) d s\right) .
$$


The right hand side of $(8.9)$ is computed as follows. Since $D_{s} w(u)=1$ if $s \leqq u,=0$ if $s>u$, we have

$$
D_{s} H_{u}= \begin{cases}\frac{\alpha\left(e_{u}(w)\right)^{2}\left[e_{1}(w)+2 \alpha\left(A_{1}(w)-A_{s}(w)\right)\right]}{\left(e_{1}(w)+\alpha\left(A_{1}(w)-A_{u}(w)\right)\right)^{2}}, & s>u, \\ \frac{-\alpha e_{1}(w)\left(e_{u}(w)\right)^{2}}{\left(e_{1}(w)+\alpha\left(A_{1}(w)-A_{u}(w)\right)\right)^{2}}, & s \leqq u,\end{cases}
$$

and

$$
\left(D^{+} H\right)_{s}+\left(D^{-} H\right)_{s}=\frac{2 \alpha^{2}\left(A_{1}(w)-A_{s}(w)\right)\left(e_{s}(w)\right)^{2}}{\left(e_{1}(w)+\alpha\left(A_{1}(w)-A_{s}(w)\right)\right)^{2}},
$$

which gives

$$
\begin{aligned}
\left|d_{c}(D H)\right| & =\exp \left(\alpha^{2} \int_{0}^{1} \frac{\left(e_{s}(w)\right)^{2}\left(A_{1}(w)-A_{s}(w)\right)}{\left(e_{1}(w)+\alpha\left(A_{1}(w)-A_{s}(w)\right)\right)^{2}} d s\right) \\
& =\left(1+\alpha Z_{1}(w)\right) \exp \left(-\frac{\alpha Z_{1}(w)}{1+\alpha Z_{1}(w)}\right) .
\end{aligned}
$$

We end this section by giving further remarks on the Ramer-Kusuoka formula, the Buckdahn-Föllmer formula and our results.

Remark 8.2. The comparison of (1.12) and (1.13) provides the following expression for the Carleman-Fredholm determinant:

$$
\left|d_{c}(D H)\right|=\left(1+\int_{0}^{1} k_{s}^{\prime}\left(w, \eta_{1}(w)\right) d s\right)^{-1} \exp \left(\int_{0}^{1}\left(D_{-} H\right)_{s} d s\right)
$$

since the two stochastic integrals in (1.12) and (1.13) satisfy

$$
\int_{0}^{1} H_{s} d w(s)=\delta(H)+\int_{0}^{1}\left(D_{-} H\right)_{s} d s,
$$

where, on the left hand side, the stochastic integral is obtained by enlargement of filtration and can be identified with the forward integral (see [19], [15]). Formula (8.11) coincides with the expression given in (8.10).

Remark 8.3. We recall that Buckdahn and Föllmer have obtained (1.13) by using a conditional Girsanov transformation, that is, the behavior of the solution (1.11) is studied under the law of a Brownian bridge ending at $y$. Then, by identification with the Ramer-Kusuoka formula (1.12), they deduced the explicit formula (8.11). In our particular case, we would like to compute directly the Carleman-Fredholm determinant to give another proof of (1.6). 
Note that, in our case, we also have a Ramer-Kusuoka formula for the density $d P_{\eta} / d P$ :

$$
\frac{d P_{\eta}}{d P}=\left|d_{c}(D K)\right| \exp \left(\delta(K)-\frac{1}{2} \int_{0}^{1} K_{s}^{2} d s\right),
$$

where

Since

$$
K_{s}=\frac{\alpha\left(e_{s}(w)\right)^{2}}{e_{1}(w)+\alpha A_{s}(w)}
$$

$$
\frac{d P_{\eta}}{d P}=\left(\frac{d P_{\xi}}{d P}\right)^{-1} \circ \xi
$$

and the exponent on the right hand side of (8.12) is easily computed as before, we obtain

$$
\left|d_{c}(D H)\right|\left|d_{c}(D K)\right| \circ \xi=\left(1+\alpha Z_{1}(w)\right)^{2} \exp \left(-\frac{2 \alpha Z_{1}(w)}{1+\alpha Z_{1}(w)}\right),
$$

with

$$
D_{s} K_{u}= \begin{cases}-\frac{\alpha\left(e_{u}(w)\right)^{2} e_{1}(w)}{\left(e_{1}(w)+\alpha A_{u}(w)\right)^{2}}, & s>u, \\ \frac{\alpha\left(e_{u}(w)\right)^{2}\left(e_{1}(w)+2 \alpha^{2} A_{s}(w)\right)}{\left(e_{1}(w)+\alpha A_{u}(w)\right)^{2}}, & s<u .\end{cases}
$$

Note the similarity of the two expressions for $D K$ and $D H$. In order to deduce the value of $\left|d_{c}(D K)\right|$ and $\left|d_{c}(D H)\right|$ from (8.13), it would be enough to prove that $\left|d_{c}(D K)\right|=\left|d_{c}(D H)\right|$ holds and that these quantities are invariant under the transform $\xi$ (this is indeed the case since, by $(8.10),\left|d_{c}(D H)\right|$ is a function of $Z_{1}$ ). Unfortunately, we have not succeeded in proving this fact.

Remark 8.4. The Ramer-Kusuoka formula is a powerful tool to study the Markov field property of the solutions of stochastic differential equations with boundary conditions (i.e., a relation between the initial value and the final value at time 1). For example, see [5] and [16]. In these works, the CarlemanFredholm determinant may be computed explicitly, using the following series expansion which involves the finite dimensional determinants:

$$
d_{c}(A)=1+\sum_{n=2}^{\infty} \frac{(-1)^{n}}{n !} \int_{[0,1]^{n}} \operatorname{det}\left(\hat{A}\left(s_{i}, s_{j}\right)\right) d s_{1} \cdots d s_{n},
$$

where $\hat{A}\left(s_{i}, s_{j}\right)=A\left(s_{i}, s_{j}\right)$ if $i \neq j$ and $\hat{A}\left(s_{i}, s_{i}\right)=0$. In formula (8.14), we have identified the Hilbert-Schmidt operator on $L^{2}([0,1])$ with a square integrable integral kernel. We have not succeeded in computing $\left|d_{c}(D H)\right|$ for the transform (8.1) from the expression (8.14). 


\section{$\S 9 . \quad$ Concluding Remarks}

\subsection{Transforms of Brownian motion with no loss of information.} In this subsection we present a number of transforms of geometric Brownian motion $\exp \left(B_{t}\right)$ which have the same filtrations as $\mathcal{B}_{t} \equiv \sigma\left\{B_{s}, s \leqq t\right\}$. We assume $\mu=0$ for simplicity.

Proposition 9.1. For every $m<3 / 4$, the stochastic process $\mathcal{E}^{(m)}=$ $\left\{\mathcal{E}_{t}^{(m)}, t \geqq 0\right\}$ given by

$$
\mathcal{E}_{t}^{(m)}=\exp \left(B_{t}\right)\left(A_{t}\right)^{-m}
$$

has the same filtration as $\left(\mathcal{B}_{t}\right)$.

Remark 9.1. Recall that $\langle\exp (B)\rangle_{t}=\int_{0}^{t} \exp \left(2 B_{s}\right) d s \equiv A_{t}$. Hence, $\mathcal{E}^{(m)}$ represents the process $\left\{\exp \left(B_{t}\right)\right\}$ "normalized" by a power of its bracket (i.e., "intrinsic time").

Proof. In [13] Proposition 7.1, we have shown the following: for any $c<3 / 2$, the filtration of the process $\left\{\exp \left(-B_{t}\right) \int_{0}^{t} \exp \left(c B_{s}\right) d s, t \geqq 0\right\}$ is the same as $\left(\mathcal{B}_{t}\right)$. From the scaling argument, this is equivalent to the filtration of $\left\{\exp \left(-2 B_{t} / c\right) A_{t}, t \geqq 0\right\}$ being the same as $\left(\mathcal{B}_{t}\right)$ for any $c<3 / 2$; equivalently, the filtration of $\left\{\exp \left(-B_{t}\right)\left(A_{t}\right)^{c / 2}, t \geqq 0\right\}$ is the same as $\left(\mathcal{B}_{t}\right)$. Hence we obtain the statement of the proposition.

We now extend the validity of Proposition 9.1. by replacing the function $x \mapsto x^{m}$ by a more general increasing function.

Proposition 9.2. Let $f:(0, \infty) \rightarrow(0, \infty)$ be continuous and increasing, and assume that $f(x)^{-2}$ is integrable near $x=0$. Then, the filtration of the process $\left\{\mathcal{E}_{t}^{(f)}, t>0\right\}$ given by

$$
\mathcal{E}_{t}^{(f)}=\exp \left(B_{t}\right)\left(f\left(A_{t}\right)\right)^{-1}, \quad t>0
$$

is equal to $\left(\mathcal{F}_{t}\right)$.

Proof. Note that $\int_{0}^{t}\left(\mathcal{E}_{s}^{(f)}\right)^{2} d s=\int_{0}^{A_{t}}(f(x))^{-2} d x$ is adapted to the natural filtration of $\mathcal{E}^{(f)}$; hence, so is $A_{t}$ and, finally, $B_{t}$.

9.2. Another discussion. A different kind of discussion consists in looking at the filtration of the stochastic process defined by

$$
Z_{t}^{\varphi, H} \equiv \varphi\left(B_{t}\right)\left(H_{t}(B)\right)^{-1}, \quad t>0,
$$

for a regular function $\varphi$ and an adapted, increasing continuous process $\left\{H_{t}(B)\right.$, $t \geqq 0\}$. 
Proposition 9.3. Denote by $\mathcal{T}$ the class of $C^{1}$ functions $\varphi: \mathbf{R} \rightarrow \mathbf{R}$ which are strictly increasing and such that $\varphi / \varphi^{\prime}$ is injective. Then, for any $\varphi \in \mathcal{T}$, the natural filtration of $\left\{Z_{t}^{\varphi, H}, t \geqq 0\right\}$ is identical to $\left(\mathcal{B}_{t}\right)$ for all $\varphi \in \mathcal{T}$.

Proof. Note that

$$
\left\langle Z^{\varphi, H}\right\rangle_{t}-\left\langle Z^{\varphi, H}\right\rangle_{s}=\int_{s}^{t}\left(\frac{\varphi^{\prime}\left(B_{u}\right)}{H_{u}(B)}\right)^{2} d u .
$$

Hence, the process $\left\{\varphi^{\prime}\left(B_{u}\right)\left(H_{u}(B)\right)^{-1}, u>0\right\}$ is adapted to the filtration of $\left\{Z_{t}^{\varphi, H}\right\}$, and so is $\left\{\left(\varphi / \varphi^{\prime}\right)\left(B_{u}\right), u \geqq 0\right\}$. The result now follows from the injectivity of $\varphi / \varphi^{\prime}$.

Examples of functions $\varphi$ belonging to $\mathcal{T}$ are: $\varphi_{1}(x)=\exp (a x)+b(a>$ $0, b \neq 0), \varphi_{2}(x)=\sinh (a x)(a>0), \varphi_{3}(x)=x^{2 k-1}(k \in \mathbf{N})$, whereas obviously the function of most interest to us, $\varphi_{0}(x)=\exp (a x)(a>0)$, does not belong to $\mathcal{T}$.

\section{References}

[1] Beneš, V. E., Nonexistence of strong nonanticipating solutions to stochastic DE's: implications for functional DE's, filtering, and control, Stoch. Proc. Appl., 5 (1977), 243-263.

[2] - Realizing a weak solution on a probability space, Stoch. Proc. Appl., 7 (1978), $205-225$.

[3] , Realizing a weak solution on a probability space, in Measure Theory Application to Stochastic Analysis, Ed. by G. Kallianpur and D. Kölzow, 101-113, Lec. Notes in Math., 695, Springer-Verlag, 1978.

[4] Buckdahn, R. and Föllmer, H., A conditional approach to the anticipating Girsanov transformation, Prob. Theory. Relat. Fields, 95 (1993), 311-330.

[5] Donati-Martin, C., Equations différentielles stochastiques dans R avec conditions aux bords, Stochastics Stochastics Rep., 35 (1991), 143-173.

[6] Dufresne, D., The distribution of a perpetuity, with application to risk theory and pension funding, Scand. Actuar. J., No.1-2 (1990), 39-79.

[7] $\longrightarrow$, An affine property of the reciprocal Asian option process, Osaka J. Math., 38 (2001), 379-381.

[8] Föllmer, H. and Imkeller, P., Anticipation cancelled by a Girsanov transformation: a paradox on Wiener space, Ann. Inst. H. Poincaré, Prob. Stat., 29 (1993), 569-586.

[9] Kusuoka, S., The nonlinear transformation of Gaussian measures on Banach space and its absolute continuity, J. Fac. Sci., Univ. Tokyo Sect. IA, 29 (1982), 567-597.

[10] Liptser, R. S. and Shiryayev, A. N., Statistics of Random Processes I, general Theory, 2nd Ed., Springer-Verlag, Berlin, 2001.

[11] Matsumoto, H. and Yor, M., A version of Pitman's $2 M-X$ theorem for geometric Brownian motions, C. R. Acad. Sc. Paris, 328 (1999), 1067-1074.

[12] - A Relationship between Brownian motions with opposite drifts via certain enlargements of the Brownian filtration, Osaka J. Math., 38 (2001), 383-398.

[13] - An analogue of Pitman's $2 M-X$ theorem for exponential Wiener functionals, Part I: A time-inversion approach, Nagoya Math. J., 159 (2000), 125-166. 
[14] Matsumoto, H. and Yor, M., An analogue of Pitman's $2 M-X$ theorem for exponential Wiener functionals, Part II: the role of the generalized inverse Gaussian laws, Nagoya Math. J., 162 (2001), 65-86.

[15] Nualart, D., The Malliavin Calculus and Related Topics, Springer-Verlag, Berlin, 1995.

[16] Nualart, D. and Pardoux, E., Boundary value problems for stochastic differential equations, Ann. Prob., 19 (1991), 1118-1144.

[17] Ramer, R., On nonlinear transformations of Gaussian measures, J. Func. Anal., 15 (1974), 166-187.

[18] Revuz, D. and Yor, M., Continuous Martingales and Brownian Motion, 3rd. Ed., Springer-Verlag, Berlin, 1999.

[19] Russo, E. and Vallois, P., Forward, backward and symmetric stochastic integration, Prob. Th. Rel. Fields, 97 (1993), 403-421.

[20] Yor, M., Sur certaines fonctionnelles exponentielles du mouvement brownien réel, $J$. Appl. Prob., 29 (1992), 202-208. 